\title{
Lista de espécies de Phlebotominae (Diptera, Psychodidae) do Estado de São Paulo, Brasil, com comentários sobre sua distribuição geográfica
}

\author{
Paloma Helena Fernandes Shimabukuro ${ }^{1,2,4}$ \& Eunice Aparecida Bianchi Galati ${ }^{3}$ \\ ${ }^{1}$ Programa de Pós-Graduação em Ciências, Coordenadoria de Controle de Doenças, \\ Secretaria Estadual de Saúde, Av. Dr. Arnaldo, n. 355, Cerqueira César, São Paulo, SP, Brasil \\ ${ }^{2}$ Instituto Leônidas e Maria Deane, Fundação Oswaldo Cruz-Amazônia, \\ Rua Terezina, n. 476, Adrianópolis, CEP 69057-070, Manaus, AM, Brasil \\ ${ }^{3}$ Departamento de Epidemiologia, Faculdade de Saúde Pública, Universidade de São Paulo - USP, \\ Av.Dr. Arnaldo,n. 755, Cerqueira César, São Paulo, SP, Brasil, e-mail: egalati@usp.br \\ ${ }^{4}$ Autor para correspondência: Paloma Helena Fernandes Shimabukuro,e-mail: phfs@yahoo.com
}

SHIMABUKURO, P.H.F. \& GALATI, E.A.B. Checklist of Phlebotominae (Diptera, Psychodidae) from São Paulo State, Brazil, with notes on their geographical distribution. Biota Neotrop 11(1a): http://www. biotaneotropica.org.br/v11n1a/en/abstract?inventory+bn0361101a2011.

\begin{abstract}
Phlebotomine sand flies are medically important insects, responsible for the transmission of Leishmania parasites between humans and non-human animal reservoirs, which are found throughout São Paulo State, Brazil. The 69 recorded species of phlebotomine sand flies from São Paulo State, including 7 species reported here for the first time to occur in this region, are organized in a checklist using the phylogenetic classification of Galati (2003). Our checklist incorporates and updates those previously published by Barretto (1947) and Martins et al. (1978), and includes records for 33 additional phlebotomine species taken from the literature published since and our examination of specimens held in entomological museum collections. For each sand fly species, the geographical distribution by municipality is also given, together with comments on the distribution of the six vectors of cutaneous leishmaniasis, as well as Lutzomyia longipalpis, the main vector of visceral leishmaniasis.

Keywords: Phlebotominae, biodiversity of the State of São Paulo, BIOTA/FAPESP Program, distribution, epidemiology, leishmaniasis, vectors.
\end{abstract}

Number of species: in the world: 927, in Brazil: 260, estimated in São Paulo State: 75.

SHIMABUKURO, P.H.F. \& GALATI, E.A.B. Checklist dos Phlebotominae (Diptera, Psychodidae) do Estado de São Paulo, Brasil, com comentários sobre sua distribuição geográfica. Biota Neotrop. 11(1a): http://www. biotaneotropica.org.br/v11n1a/pt/abstract?inventory+bn0361101a2011.

Resumo: Os flebotomíneos são insetos de interesse médico, responsáveis pela transmissão de protozoários parasitas do gênero Leishmania entre animais reservatórios humanos e não-humanos, os quais são encontrados em todo o Estado de São Paulo, Brasil. As 69 espécies de flebotomíneos registradas no Estado de São Paulo, incluindo 7 espécies registradas aqui pela primeira vez, estão organizadas em uma lista de espécies utilizando a classificação filogenética de Galati (2003). Nossa lista de espécies incorpora e atualiza os dados publicados por Barretto (1947) e Martins et al. (1978), e inclui registros para mais 33 espécies retirados da literatura publicada desde então, e também da observação de espécimes depositados em coleções entomológicas. Para cada espécie de flebotomíneo, a distribuição geográfica por município é fornecida, com comentários sobre a distribuição de seis vetores de leishmaniose cutânea, bem como de Lutzomyia longipalpis, o principal vetor de leishmaniose visceral. Palavras-chave: Phlebotominae, biota paulista, Programa BIOTA/FAPESP, distribuição, epidemiologia, leishmanioses, vetores.

Número de espécies: no mundo: 927, no Brasil: 260, estimadas no Estado de São Paulo: 75. 


\section{Introdução}

Os flebotomíneos são vetores de protozoários parasitas do gênero Leishmania, os quais são transmitidos em ciclos silváticos ou peridomésticos entre o homem e animais domésticos e silvestres, pela picada do inseto vetor (Rangel \& Lainson 2003). As leishmanioses são doenças com crescente importância epidemiológica por causa da sua urbanização (Desjeux 2004). No Estado de São Paulo (SP), Brasil, foram registrados 7.512 casos de leishmaniose cutânea (LC) entre 1998 e 2009 (Centro... 1998a), e mais de 1.628 casos de leishmaniose visceral (LV) entre 1999 e 2009 (Centro... 1998b).

Mais de 927 espécies ou subespécies de flebotomíneos atuais e 22 fósseis foram descritas, sendo que aproximadamente 500 atuais e 16 fósseis ocorrem no Novo Mundo. No Estado de São Paulo, 69 espécies foram registradas (Galati 2003, Galati et al. 2007, Odorizzi e Galati 2007, Shimabukuro et al. 2007), incluindo os principais vetores de leishmaniose cutânea (Nyssomyia intermedia sensu lato e $N$. whitmani) e leishmaniose visceral (Lutzomyia longipalpis). Das 19 espécies suspeitas ou incriminadas de transmitir Leishmania spp. que infectam humanos registradas no Brasil, seis são encontradas no Estado de São Paulo, além das três espécies mencionadas acima: Migonemyia migonei, Pintomyia fischeri e P. pessoai (Rangel \& Lainson 2003).

Checklists regionais e listas de faunas são importantes fontes de informação taxonômica, biogeográfica e biológica, pois auxiliam na identificação correta das espécies, na detecção de erros nas etiquetas dos espécimes e permitem inferências acerca da distribuição de outras espécies com conhecida interação (i.e. associações entre parasitas e hospedeiros, em que a distribuição dos primeiros determina a distribuição dos últimos) (Cranston 2005). Infelizmente, a compilação e publicação de listas de espécies geralmente são consideradas de pouca importância e pouco atraentes (Cranston 2005). Entretanto, a informação fornecida pelos checklists e listas de fauna auxilia em diversos aspectos da entomologia aplicada, como em epidemiologia, e no manejo e controle de pestes/vetores (Civelek 2003, Reinert et al. 2005).

Diversos catálogos e trabalhos contendo dados de distribuição geográfica de flebotomíneos, incluindo aqueles do Estado de São Paulo, foram publicados na primeira metade do século 20 (Costa Lima 1932, Barretto 1947). Barretto (1947) publicou um catálogo dos flebotomíneos americanos que incluiu 32 espécies coletadas no Estado de São Paulo, além de uma lista com a distribuição das espécies por municípios. A publicação mais recente descrevendo a distribuição de flebotomíneos no Estado de São Paulo por municípios foi a de Martins et al. (1978), na qual 36 espécies foram registradas (as 32 espécies originalmente catalogadas por Barretto (1947), mais 4 novas espécies registradas). Nenhuma lista atualizada para as espécies de flebotomíneos do Estado São Paulo foi publicada desde então. Os trabalhos de Barretto (1947) e Martins et al. (1978) foram baseados em sistemas de classificação artificiais que não refletem as relações evolutivas dos flebotomíneos. No presente trabalho, apresentamos um checklist atualizado das espécies de flebotomíneos registradas até o momento no Estado de São Paulo, segundo os municípios, utlizando a classificação filogenética, baseada em caracteres morfológicos, proposta por Galati (2003).

\section{Materiais e Métodos}

Área de estudo, Estado de São Paulo, Brasil, tem uma área total de 248.209.426 km², a qual compreende 645 municípios, com uma população total de aproximadamente 39.827 .570 ha (Instituto... 2010).

A lista de espécies e os dados de distribuição geográfica compilados neste trabalho foram obtidos a partir: i) da literatura publicada, incluindo os checklists previamente publicados por
Barretto (1947) e Martins et al. (1978); ii) do nosso exame de espécimes depositados nas coleções entomológicas do Museu de Zoologia, Universidade de São Paulo (MZUSP), e da Faculdade de Saúde Pública, Universidade de São Paulo (FSP); iii) dos nossos próprios dados não publicados, resultantes de coletas entomológicas; e iv) da comunicação pessoal com o Dr. Claudio Casanova (SUCEN/ SES-SP, Superintendência de Controle de Endemias/Secretarias de Saúde do Estado de São Paulo), resultantes de coleta entomológica para vigilância e controle de vetores.

Para os registros de espécies publicados nos trabalhos de Barretto (1947) e Martins et al. (1978) inclusos em nosso checklist, citamos estas duas referências ao invés das fontes originais, para facilitar a identificação das espécies que foram citadas previamente nestes dois trabalhos. Os trabalhos de Barretto (1947) e Martins et al. (1978) devem portanto, ser consultados para informações mais detalhadas acerca dos registros das espécies e das localidades. No total, examinamos 21 espécies de flebotomíneos, de 41 localidades diferentes, provenientes do material depositado nas coleções entomológicas. Quando a informação sobre a distribuição geográfica de uma espécie em nosso checklist é proveniente do exame deste material, a abreviação da instituição a qual o material se encontra depositado é dada entre parênteses, e.g. (MZUSP) ou (FSP).

Para garantir consistência em relação à classificação taxonômica, espécimes identificados por outros taxonomistas usando classificações anteriores (Forattini 1973, Young \& Duncan 1994) foram reexaminados e reclassificados de acordo com Galati (2003). Sinonímias podem ser encontradas nos trabalhos de Martins (1978) e Galati (2003). As localidades-tipo estão sublinhadas e o sexo da espécie-tipo é dado entre colchetes (e.g. [ ]).

\section{Resultados e Discussão}

Pelo menos uma das quatro fontes listadas nos Materiais e Métodos demonstrou a ocorrência das 69 diferentes espécies de flebotomíneos (Apêndice 1) registradas no Estado de São Paulo. As fontes incluídas em nosso checklist datam de 1943 a 2010, e descrevem a ocorrência de pelo menos uma espécie de flebotomíneo em 210 (32\%) dos 645 municípios do Estado de São Paulo.

Nosso checklist inclui todos os registros para as espécies registradas nos trabalhos de Barretto (1947) e Martins et al. (1978), ainda que reclassificados de acordo com Galati (2003)(ver mais adiante, para maiores detalhes). Além das 36 espécies previamente catalogadas por Barretto (1947) e/ou Martins et al. (1978), encontramos registros para mais 33 espécies de flebotomíneos não incluídas em trabalhos anteriores desta subfamília no estado de São Paulo. Os registros para 26 dessas espécies adicionais de flebotomíneos são procedentes da literatura publicada desde os trabalhos de Barretto (1947) e Martins et al. (1978). As outras 7 espécies adicionais foram coletadas por uma de nós (E.A.B. Galati) e pelo Dr. Claudio Casanova, e não foram publicadas anteriormente. Estas 7 espécies são novas ocorrências para o Estado de São Paulo: Brumptomyia bragai, Micropygomyia quinquefer, Lutzomyia almerioi, Pintomyia christenseni, Evandromyia carmelinoi, E. rupicola, e Psychodopygus hirsutus.

Suspeitamos de identificações errôneas em relação a Pressatia choti e $P$. trispinosa, registradas, respectivamente, por Forattini et al. (1976b) e Barretto (1943). Tais suspeitas fundamentam-se no fato de que as fêmeas das espécies do gênero Pintomyia christenseni e P. mamedei são parecidas às do gênero Pressatia e machos deste gênero nunca foram coletados no Estado de São Paulo.

Nosso checklist inclui as seis espécies de flebotomíneos implicadas na transmissão de LC no Estado de São Paulo. O número de municípios em que estas espécies foram registradas é dado entre 
parênteses: $N$. intermedia (13), N. neivai (40), N. whitmani (105), P. fischeri (121), P. pessoai (69) e M. migonei (93). O principal vetor L. infantum ( = L. chagasi), agente etiológico da LV, L. longipalpis, foi registrado em 29 municípios do Estado de São Paulo.

Neste trabalho, apresentamos pela primeira vez um checklist para as 69 espécies de flebotomíneos registradas no Estado de São Paulo. Nosso checklist incorporou as 36 espécies catalogadas anteriormente por Barretto (1947) e Martins et al. (1978), e incluiu registros para 33 espécies adicionais de flebotomíneos nunca publicadas anteriormente para o estado de São Paulo. Além do mais, nosso checklist atualizou os dados de Barretto (1947) e Martins et al. (1978) reclassificando as espécies reportadas em seus trabalhos, de acordo com a classificação filogenética mais recente de Galati (2003). Galati (2003) reagrupou as espécies de flebotomíneos americanos em 22 gêneros, dos quais 14 foram registrados no estado de São Paulo. Quando Barretto (1947) publicou seu catálogo de flebotomíneos americanos, ele incluiu todas as 156 espécies conhecidas até então, em um único gênero Flebotomus, que não é mais reconhecido. Assim, todas as espécies catalogadas por Barretto (1947) como pertencendo ao gênero Flebotomus, foram reclassificadas em nosso checklist em 9 gêneros diferentes (Brumptomyia, Lutzomyia, Migonemyia, Pintomyia, Evandromyia, Psathyromyia, Martinsmyia, Psychodopygus, Nyssomyia). Martins et al. (1978) atualizou o trabalho de Barretto (1947), e listou as 354 espécies do Novo Mundo, dividas em 4 gêneros (Brumptomyia, Warileya, Hertigia e Lutzomyia). Destes, somente os gêneros Brumptomyia e Lutzomyia tinham sido registrados para o estado de São Paulo. O gênero Brumptomyia se manteve na classificação de Galati (2003), mas Lutzomyia foi dividido em 18 gêneros, dos quais 11 ocorrem no estado de São Paulo (Micropygomyia, Sciopemyia, Lutzomyia, Migonemyia, Pintomyia, Expapillata, Evandromyia, Psathyromyia, Martinsmyia, Psychodopygus e Nyssomyia).

Este trabalho também inclui a distribuição das espécies de flebotomíneos no estado de São Paulo por município, para auxiliar as atividades de vigilância e controle entomológico de vetores de LC e LV. Entretanto, nosso checklist não deve ser considerado exaustivo, pois o número de espécies flebotomíneos registrado continua a crescer, conforme novas espécies são descritas ou registradas pela primeira vez para a região, especialmente como resultado do trabalho entomológico desenvolvido pela SUCEN/SES-SP dentro do Programa de Vigilância e Controle das Leishmanioses do estado de São Paulo. Os registros de distribuição geográfica para as espécies que listamos refletem resultados de áreas nas quais a maior parte das coletas entomológicas foi realizada, primariamente por causa da importância epidemiológica destas áreas (i.e. áreas de transmissão de leishmanioses, maior densidade populacional e/ou uso intensivo da terra). Os flebotomíneos, portanto, não estão necessariamente ausentes daqueles municípios em que não há registros ou há registros de poucas espécies, já que isso pode ser um reflexo do escasso trabalho entomológico realizado nessas áreas. Assim, entre as espécies registradas mais comuns encontradas no estado de São Paulo estão os vetores suspeitos ou incriminados de leishmanioses, principalmente LC. De maneira geral, há evidência de que pelo menos uma das sete espécies suspeitas ou incriminadas de LC ou LV ocorre em 195 (30.2\%) dos municípios de São Paulo. Para os 450 municípios restantes, não foi possível localizar registros de nenhuma das sete espécies de vetores suspeitos ou incriminados de leishmanioses. Nos municípios sem registros de flebotomíneos, estes insetos podem ter sido pesquisados, mas não encontrados, ou a coleta entomológica foi inapropriada ou os resultados nunca foram publicados formalmente. Embora não tenha sido possível encontrar evidência da ocorrência de flebotomíneos nestes locais, é possível que uma ou mais das sete espécies de vetores de leishmanioses: L. longipalpis, M. migonei, $N$. intermedia, $N$. neivai (considerada sinônimo júnior de
$N$. intermedia até ser revalidada por Marcondes 1996), N. whitmani, P. fischeri e P. pessoai, possa(m) ocorrer em alguns destes municípios.

A aparente introdução recente e expansão geográfica de L. longipalpis em área urbana de Araçatuba e cidades vizinhas (Costa et al. 1997, Camargo-Neves et al. 2003) fornece um exemplo de como a distribuição dos flebotomíneos é dinâmica, e da necessidade contínua de vigilância entomológica. Padrões de transmissão de LC e LV no estado de São Paulo têm mudado devido ao impacto antrópico no meio ambiente. Fatores que contribuem para a emergência de doenças transmitidas por vetores incluem mudanças no uso da terra - associados à urbanização, migração e mobilidade das populações, além da construção de represas, gasodutos e estradas (Vasconcelos et al. 2001) - os quais podem causar redistribuição da fauna sinantrópica (Cranston 2005). Novas ferramentas para melhorar a vigilância dos casos humanos e de reservatórios (silvestres e domésticos) de leishmanioses, assim como o monitoramento da distribuição das populações de insetos vetores, precisam ser desenvolvidas. Potanto, a informação fornecida por dados de distribuição descritivos e checklists pode fornecer um ponto de partida para gerar informações mais sofisticadas e mapas preditivos de risco para doenças e distribuição de vetores baseados em métodos estatísticos (Randolph 2000).

\section{Principais grupos de pesquisa}

- Parasitoses transmitidas por vetores: aspectos epidemiológicos e diagnósticos.

Líderes: José Eduardo Tolezano, Vera Lucia Pereira-Chioccola

- Estudo de flebotomíneos do cerrado Pantanal. Líderes: Alessandra Gutierrez de Oliveira, Maria Elizabeth Moraes Cavalheiros Dorval.

- Taxonomia de flebotomíneos/Epidemiologia, diagnóstico e controle das leishmanioses.

Líderes: Edelberto Santos Dias, Celia Maria Ferreira Gontijo

- Ecologia das Leishmanioses. Líderes: Elizabeth Ferreira Rangel, Maurício Luiz Vilela.

\section{Principais acervos}

Existem no Brasil, quatro principais coleções que abrigam espécimes de flebotomíneos (Apêndice 2):

- Coleção de Invertebrados (MZUSP), esta coleção abrigou parte das lâminas que pertenceram à Coleção Padrão do Departamento de Parasitologia, Faculdade de Medicina/USP, organizada pelo Dr. Mauro Pereira Barretto ao longo de quase 60 anos de pesquisa;

- Coleção de Entomologia Médica do Departamento de Epidemiologia da Faculdade de Saúde Pública (USP), esta coleção abrigou a outra parte das lâminas que pertenceram à Coleção Padrão do Departamento de Parasitologia, Faculdade de Medicina/USP, organizada pelo Dr. Mauro Pereira Barretto;

- Coleção de Referência Internacional e Nacional de Flebotomíneos (Centro de Pesquisas René Rachou/FIOCRUZ);

- Coleção Entomológica do Instituto Oswaldo Cruz (FIOCRUZ), coleção iniciada em 1901 por Oswaldo Cruz, abriga a coleção histórica "Otávio Mangabeira" que contém 1632 flebotomíneos identificados até o nível de espécie.

\section{Principais lacunas do conhecimento}

A leishmaniose cutânea é conhecida no Estado de São Paulo desde pelo menos 1884 (Tolezano 1994). A transmissão desta doença aumentou como consequência da expansão das plantações de café no século 19 e continuou nas primeiras décadas do século 20 , conforme as fazendas de café foram se estabelecendo no noroeste do 
estado, e estradas de trem foram construídas para escoar a produção (Tolezano 1994). Foi nas primeiras décadas do século 20 que a fauna de flebotomíneos começou a ser estudada por Lutz, Barretto e Coutinho, entre outros pesquisadores, ampliaram o conhecimento sobre a biologia, a epidemiologia e principalmente a taxonomia com a descrição de diversas novas espécies.

A re-emergência da LC (Camargo-Neves et al. 2002) e emergência da LV (Galimbertti et al. 1999) no Estado de São Paulo trouxe um novo interesse nos estudos dos flebotomíneos, e técnicas moleculares passaram a fazer parte dos estudos epidemiológicos (Rangel \& Lainson 2003, Paiva et al. 2004).

Apesar da grande quantidade de estudos epidemiológicos e biológicos, o avanço em relação à taxonomia e sistemática dos flebotomíneos americanos foi mais lento. A sistemática desta subfamília é controversa, especialmente em nível supra-específico. Uma abordagem conservadora baseada em critérios práticos (Lewis et al. 1977) dividiu os flebotomíneos em cinco gêneros, dos quais três (Brumptomyia, Warileya e Lutzomyia) são representados no Novo Mundo, e, estão distribuídos em mais de 480 espécies (Galati 2003).

Artemiev (1991) defendeu uma classificação natural e dividiu os flebotomíneos em duas tribos e sete subtribos, mesmo assim, não estudou mais detalhadamente os flebotomíneos americanos e, sua classificação não foi aceita entre os pesquisadores desse grupo de insetos.

Uma única proposta para a sistemática dos Phlebotominae baseada no método cladístico, foi apresentada por Galati (1995, 2003), que manteve as duas tribos de Artemiev (1991), criou oito subtribos, e classificou os flebotomíneos em 22 gêneros, dos quais 14 ocorrem no Estado de São Paulo. Em 2003, um estudo conduzido por Beati et al. 2003 baseado em análise de sequências de DNA ribossomal 12s e 28s de espécies andinas do Peru confirmaram a divisão de Galati em pelo menos dois clados que correspondem às duas subtribos (Lutzomyiina e Psychodopygina).

Assim, como principais lacunas do conhecimento, identificamos a necessidade de:

- Estudos das relações filogenéticas dos flebotomíneos em níveis de gênero e espécie, utilizando-se caracteres morfológicos e moleculares;

- Estudos de genética de populações de flebotomíneos, especialmente nos casos de espécies crípticas;

- Desenvolvimento de marcadores para DNA barcoding, principalmente para as espécies de interesse médico;

- Sistematização dos dados de distribuição geográfica para permitir a construção de mapas preditivos.

\section{Perspectivas de pesquisa para os próximos 10 anos}

Novas pesrpectivas para a pesquisa surgirão com o sequenciamento completo do genoma de Lutzomyia longipalpis (VectorBase), principal vetor do agente etiológico da leishmaniose visceral na América, possibilitando a ampliação do conhecimento em genômica funcional, proteômica e filogenômica. Estes estudos poderão contribuir para o esclarecimento das relações filogenéticas, da biogeografia, além da prospecção de moléculas-alvo para o desenvolvimento de vacinas de bloqueio de transmissão. Há também, a perspectiva de desenvolvimento de sequências para DNA barcoding que poderão facilitar a identificação das espécies, inclusive facilitando os trabalhos de detecção molecular de Leishmania nestes insetos

\section{Agradecimentos}

Agradecemos ao Dr. Carlos E. Lamas e Dra. Maria Anice M. Sallum, curadores, respectivamente, da Coleção de Invertebrados, Museu de Zoologia da Universidade de São Paulo (MZUSP) e
Coleção Entomológica da Faculdade de Saúde Pública - Universidade de São Paulo (FSP/USP), por permitirem acesso aos espécimes. Também agradecemos ao Dr. Claudio Casanova por compartilhar dados não publicados sobre a distribuição dos flebotomíneos coletados no estado de São Paulo, e ao Dr. Luke Baton por comentários no manuscrito.

\section{Referências Bibliográficas}

ANDRADE-FILHO, J.D., GALATI, E.A.B. \& FALCÃO, A.L. 2007. Nyssomyia intermedia (Lutz and Neiva, 1912) and Nyssomyia neivai (Pinto, 1926) (Diptera: Psychodidae: Phlebotominae) geographical distribution and epidemiological importance. Mem. Inst.Oswaldo Cruz 102(4):481-487. http://dx.doi.org/10.1590/S0074-02762007005000035

ARTEMIEV, M.M. 1991. A classification of the subfamily Phlebotominae. Parassitol 33(Suppl. 1):69-77.

BARRETTO, M.P. \& COUTINHO, J.O. 1941. Contribuição ao conhecimento dos flebótomos de São Paulo. IV. Descrição de três novas espécies. Pap. Avulsos Depart. Zool. 1(24):143-158.

BARRETTO, M.P. 1943. Observações sobre a biologia, em condições naturais, dos flebótomos do Estado de São Paulo (Diptera, Psychodidae). Tese de Livre-Docência, Universidade de São Paulo, São Paulo.

BARRETTO, M.P. 1947. Nova contribuição para o estudo da distribuição geográfica dos flebotomíneos americanos (Diptera, Psychodidae). Arch. Hig. S Públ 15:211-26.

BEATI, L., CÁCERES, A.G., LEE, J.A. \& MUNSTERMANN, L.E. 2003. Systematic relationships among Lutzomyia sandflies (Diptera: Psychodidae) of Peru and Colombia based on the analysis of $12 \mathrm{~s}$ and 28s ribosomal DNA sequences. Int J Parasitol 34(2):225-234. PMid:15037108. http://dx.doi.org/10.1016/j.ijpara.2003.10.012

BRITO, M., CASANOVA C., MASCARINI, L.M., WANDERLEY, D.M.V. \& CORRÊA, F.M.A. 2002. Phlebotominae (Diptera: Psychodidae) em área de transmissão de leishmaniose tegumentar americana no litoral norte do Estado de São Paulo, Brasil. Rev. Soc. Bras. Med. Trop. 35:431-437. PMid:12621660. http://dx.doi.org/10.1590/S0037-86822002000500002

CAMARGO-NEVES, V.L.F., GOMES, A.C. \& ANTUNES, J.L.F. 2002. Correlação da presença de espécies de flebotomíneos (Diptera: Psychodidae) com registros de casos de leishmaniose tegumentar americana no Estado de São Paulo, Brasil. Rev. Soc. Bras. Med. Trop. 35:299-306.

CAMARGO-NEVES, V.L.F., SPÍNOLA, R. \& LAGE, L. 2003. A leishmaniose visceral americana no estado de São Paulo: situação epidemiológica em 2001-2002. Rev. Soc. Bras. Med. Trop. 36(Supl. 2):27-35. PMid:12170323.

CASANOVA, C., COSTA, A.I.P. \& NATAL, D. 2005. Dispersal pattern of the sand fly Lutzomyia neivai (Diptera: Psychodidae) in a cutaneous leishmaniose endemic rural area in Southeastern Brazil. Mem. Inst. Oswaldo Cruz 100:719-724. http://dx.doi.org/10.1590/S007402762005000700006

CIVELEK, H.S. 2003. Checklist of Agromyzidae (Diptera) of Turkey, with a new record. Phytoparasitica 31(2):132-138. http://dx.doi.org/10.1007/ BF02980782

CONDINO, M.L.F., SAMPAIO, S.M.P., HENRIQUES, L.F., GALATI, E.A.B., WANDERLEY, D.M.V. \& CÔRREA, F.M.A. 1998. Leishmaniose tegumentar americana: flebotomíneos de área de transmissão no município de Teodoro Sampaio, região sudoeste do estado de São Paulo, Brasil. Rev. Soc. Bras. Med. Trop. 31(4):355-360. PMid:9662962. http://dx.doi. org/10.1590/S0037-86821998000400004

CONDINO, M.L.F., GALATI, E.A.B., HOLCMAN, M.M., SALUM, M.R.B., SILVA, D.C. \& NOVAES-JÚNIOR, R.A. 2008. American cutaneous leishmaniose on the northern coastline of the State of São Paulo, 1993 to 2005. Rev. Soc. Bras. Med. Trop. 41:635-641. PMid:19142444.

COSTA, A.I.P., CASANOVA, C., RODAS, L.A.C. \& GALATI, E.A.B. 1997. Geographical distribution and first record of Lutzomyia longipalpis in an urban area in São Paulo State, Brazil. Rev Saúde Públ. 31(6):632-633. http://dx.doi.org/10.1590/S0034-89101997000700012 
COSTA-LIMA, A. 1932. Sôbre os phlebótomos americanos (Diptera: Psychodidae). Mem. Inst.Oswaldo Cruz 26:15-69. http://dx.doi. org/10.1590/S0074-02761932000100002

CRANSTON, P. 2005. Biogeographic patterns in the evolution of Diptera. In The evolutionary biology of flies (D.K. Yeates \& B.M. Wiegmann, eds). Columbia University Press, New York, p.274-311.

CUTOLO, A.A. \& VON ZUBEN, C.J. 2008. Flebotomíneos (Diptera, Psychodidae) de área de cerrado no município de Corumbataí, centroleste do estado de São Paulo, Brasil. Rev Bras Parasitol Vet. 17(1):45-49.

CUTOLO, A.A., CAMAMARGO, D.A., CUTOLO, A.A., VON ZUBEN, C.J. \& GALATI, E.A.B. 2008. Lutzomyia longipalpis (Diptera, Psychodidae) em Cuesta Basáltica, na bacia hidrográfica do Rio Corumbataí, Região Centro-leste do Estado de São Paulo. Rev Bras Epidemiol 11(2):336-339.

CENTRO DE VIGILÂNCIA EPIDEMIOLÓGICA PROF. ALEXANDRE VRANJAC - CVE. 1998a. Leishmaniose tegumentar no Estado de São Paulo - Casos notificados por GVE e ano da notificação 2010. http://www. cve.saude.sp.gov.br/htm/zoo/lta_gve.htm (último acesso em 05/10/2010). Divisão de Zoonoses, Secretaria de Estado da Saúde.

CENTRO DE VIGILÂNCIA EPIDEMIOLÓGICA PROF. ALEXANDRE VRANJAC - CVE. 1998b. Leishmaniose visceral americana humana. Casos autóctones e óbitos de LVA, no Estado de São Paulo, 1999 a 2010. http://www.cve.saude.sp.gov.br/htm/zoo/lvah_auto9904.htm (último acesso em 05/10/2010). Divisão de Zoonoses, Secretaria de Estado da Saúde.

DESJEUX, P. 2004. Leishmaniose: current situation and new perspectives. Comp Immunol Microb 27:305-318.

DOMINGOS, M.F., CARRERI-BRUNO, G.C., CIARAVOLO, R.M.C GALATI, E.A.B., WANDERLEY, D.M.V. \& CORREA, F.M.A. 1998. Leishmaniose tegumentar americana: flebotomíneos de área de transmissão, no município de Pedro de Toledo, região sul do estado de São Paulo, Brasil. Rev. Soc. Bras. Med. Trop. 31(5):425-432.

FORATTINI, O.P. 1954. Algumas observações sobre a biologia dos flebótomos (Diptera, Psychodidae) em região da bacia do rio Paraná (Brasil). Arq. Fac. Hig. S. Paulo 8:15-136.

FORATTINI, O.P. 1973. Entomologia Médica. Psychodidae. Phlebotominae Leishmanioses. Bartonelose. Edgar Blucher Editora, São Paulo, v.4.

FORATTINI, O.P., RABELLO, E.X. \& PATOLI, D.G.B. 1970. Sobre o encontro de Lutzomyia longipalpis (Lutz and Neiva, 1912) no Estado de São Paulo, Brasil. Ver. Saúde Públ. 4:99-100.

FORATTINI, O.P., RABELLO, E.X. \& GALATI, E.A.B. 1976a. Novos encontros de flebotomíneos no Estado de São Paulo, Brasil, com especial referência a Lutzomyia longipalpis. Rev. Saúde Públ. 10:125-8.

FORATTNI, O.P., RABELLO, E.X., SERRA, O.P., COTRIM, M.D., GALATI, E.A.B. \& BARATA, J.M.S. 1976b. Observações sobre a transmissão da leishmaniose tegumentar no estado de São Paulo, Brasil. Rev. Saúde Públ. 10:31-43.

GALATI, E.A.B. 1981. Sobre a identificação de Psychodopygus (Psychodopygus) arthuri (Fonseca, 1936) e de P. (P.) lloydi (Antunes, 1937) (Diptera, Psychodidae, Phlebotominae). Rev. Bras. Entomol. 25(4):321-322.

GALATI, E.A.B. 1995. Phylogenetic systematics of Phlebotominae (Diptera: Psychodidae) with emphasis on American groups. Bol. Dir. Malariol. y Amb. 35(Suppl. 1):133-142.

GALATI, E.A.B. 2003. Classificação de Phlebotominae. In Flebotomíneos do Brasil (E.F. Rangel \& R. Lainson, eds.). Editora Fiocruz, Rio de Janeiro, p.23-52.

GALATI, E.A.B. \& GOMES, A.C. 1977. Descrição da fêmea de Lutzomyia termitophila Martins, Falcão \& Silva, 1964 (Diptera, Psychodidae, Phlebotominae). Rev. Bras. Entomol. 21(2):63-64.

GALATI, E.A.B. \& GOMES, A.C. 1992. Descrição de Lutzomyia rabelloi sp. n. (Diptera, Psychodidae, Phlebotominae) da região do Vale do Ribeira, São Paulo, Brasil. Rev Bras Entomol 36(2):457-463.

GALATI, E.A.B., NUNES, V.L.B., OSHIRO, E.T. \& REGO, F.A. 1989. Nova espécie de Phlebotominae, Lutzomyia corumbaensis, sp. n. (Diptra, Psychodidae) do complexo Lutzomyia cortelezzii. Rev Bras Entomol 33(3-4):465-475.
GALATI, E.A.B., MARASSÁ, A.M., ANDRADE, R.M.G., PINTO, P.L.S., CONSALES, C.A., KARMANN, I. \& BUENO, E.F.M. 2002. Phlebotomines from speleological province of Ribeira Valley, São Paulo State, Brasil. Entomol. Vectores (Suppl. 1):38-39.

GALATI, E.A.B., MARASSA, A.M. \& ANDRADE, R.M.G. 2003. Micropygomyia (Sauromyia) petari, a new species of phlebotominae (Diptera, Psychodidae) from Vale do ribeira, São Paulo State, Brazil. Rev. Bras. Entomol. 47(3):455-459. http://dx.doi.org/10.1590/S008556262003000300016

GALATI, E.A.B., FONSECA, M.B. \& MARASSÁ, A.M. 2007. The subgenus Migonemyia Galati 1995 (Diptera, Psychodidae, Phlebotominae), with description of a new espécies Migonemyia vaniae. Mem. Inst. Oswaldo Cruz 102(5):605-615. http://dx.doi.org/10.1590/S007402762007005000064

GALATI, E.A.B., FONSECA, M.B., MARASSÁ, A.M. \& BUENO, E.F.M. 2009. Dispersal and survival of Nyssomyia intermedia and Nyssomyia neivai (Diptera: Psychodidae: Phlebotominae) in a cutaneous leishmaniasis endemic area of the speleological province of the Ribeira Valley, state of São Paulo, Brazil. Mem. Inst. Oswaldo Cruz 104(8):1148-1158. http:// dx.doi.org/10.1590/S0074-02762009000800012

GALATI, E.A.B., MARASSÁ, A.M., GONÇALVES-ANDRADE, R.M., CONSALES, C.A. \& BUENO, E.M.F. 2010. Phlebotomines (Diptera, Psychodidae) in the Ribeira Vally speleological province - 1. Parque Estadual Intervales, state of São Paulo, Brazil. Rev. Bras. Entomol 54(2):311-321. http://dx.doi.org/10.1590/S0085-56262010000200015

GALIMBERTTI, M.Z., KATZ, G., CAMARGO-NEVES, V.L.F., RODAS, L.A.C., CASANOVA, C., COSTA, I.P., ARAUJO, M.F.L., TANIGUCHI, H.H., BARBOSA, J.A.R., BARBOSA, J.E.R., TOLEZANO, J.E. \& PINTO, P.L.S. 1999. Leishmaniose visceral americana no Estado de São Paulo. Rev. Soc. Bras. Med. Trop. 32(Suppl. 1):217-8.

GOMES, A.C, SANTOS, J.L.F. \& GALATI, E.A.B. 1986. Observations on the endophilic behaviour of the sand fly and vectorial role of Psychodopygus intermedius in the Ribeira Valley, region of the São Paulo state, Brazil Rev. Saúde Públ. 20(4):280-287.

GOMES, A.C., BARATA, J.M.S., ROCHA E SILVA, E.O. \& GALATI, E.A.B. 1989. Aspectos ecológicos da leishmaniose tegumentar americana. 6 . Fauna flebotomínea antropófila de matas residuais situadas no centronordeste do estado de São Paulo, Brasil. Rev. Ins. Med. Trop. 31(1):32-39. http://dx.doi.org/10.1590/S0036-46651989000100007

GOMES, A.C, RABELLO, E.X. \& GALATI, E.A.B. 1978. Flebotomíneos encontrados em galinheiros experimentais nos Estados de São Paulo e Minas Gerais (Brasil) e algumas observações ecológicas. Rev. Saúde Públ. 12:403-407.

GOMES, A.C. \& GALATI, E.A.B. 1987. Aspectos ecológicos da Leishmaniose tegumentar americana. 5. Estratificação da atividade espacial e estacional de Phlebotominae (Diptera, Psychodidae) em áreas da região do Vale do Ribeira, Estado de São Paulo, Brasil. Mem. Inst. Oswaldo Cruz 82 (4):467-473.

GOMES, A.C. \& GALATI, E.A.B. 1989. Aspectos ecológicos da leishmaniose tegumentar americana. 7. Capacidade vetorial flebotomínea em ambiente florestal primário do Sistema da Serra do Mar, região do Vale do Ribeira, Estado de São Paulo, Brasil. Rev. Saúde Públ. 23:136-142.

GOMES, A.C., GALATI, E.A.B. \& GLASSER, C.M. 1990a. Nota sobre encontro de Phlebotominae (Diptera: Psychodidae) no litoral sul do Estado de São Paulo, Brasil. Rev. Saúde Públ. 24:319-320.

GOMES, A.C., COUTINHO, S.G, PAIM, G.V., OLIVEIRA, S.A.O, GALATI E.A.B., NUNES, M.P., CAPINZAIKI, N., IAMAMOTO, Y.I. \& ROTTER, P. 1990b. Aspectos ecológicos da leishmaniose tegumentar americana. 8 Avaliação da atividade enzoótica de Leishmania (Viannia) braziliensis, em ambiente florestal e peridomiciliar, região do Vale do Ribeira, Estado de São Paulo, Brasil. Rev. Inst. Med. Trop. 32(2):105-115.

INSTITUTO BRASILEIRO DE GEOGRAFIA E ESTATÍSTICA - IBGE. http://www.ibge.gov.br/cidadesat/topwindow.htm?1 (ùltimo acesso em 15/01/2009).

LARROUSSE, F. 1920. Nouvelle espèce américaine du genre Phlebotomus, Phlebotomus brumpti sp. nov. Bull. Soc. Pathol. Exot. 13:659-662. 
LEWIS, D.J., YOUNG, D.G., FAIRCHILD, G.B. \& MINTER, D.M. 1977. Proposals for a stable classification of phlebotomine sandflies. Syst. Entomol. 2:319-332. http://dx.doi.org/10.1111/j.1365-3113.1977. tb00381.x

MARCONDES, C.B. 1996. A redescription of Lutzomyia (Nyssomyia) intermedia (Lutz and Neiva, 1912), and resurrection of L. neivai (Pinto, 1926) (Diptera, Psychodidae, Phlebotominae). Mem. Inst.Oswaldo Cruz 91:457-462. http:// dx.doi.org/10.1590/S0074-02761996000400012

MARCONDES, C.B., LOZOVEI, A.L. \& VILELA, J.H. 1998. Distribuição geográfica de flebotomíneos do complexo Lutzomyia intermedia (Lutz and Neiva, 1912) (Diptera, Psychodidae). Rev. Soc. Bras. Med. Trop. 31:51-58. http://dx.doi.org/10.1590/S0037-86821998000100007

MARTINS, A.V., WILLIAMS, P. \& FALCÃO, A.L. 1978. American sand flies (Dipera: Psychodidae, Phlebotominae). Academia Brasileira de Ciências, Rio de Janeiro.

MAYO, R.C., CASANOVA, C., MASCARINI, L.M., PIGNATTI, M.G., RANGEL, O., GALATI, E.A.B., WANDERLEY, D.M.V. \& CÔRREA, F M.A. 1998. Flebotomíneos (Diptera, Psychodidae) de área de transmissão de leishmaniose tegumentar americana, no município de Itupeva, região sudeste do estado de São Paulo, Brasil. Rev. Soc. Bras. Med. Trop. 31(4):339-345. http://dx.doi.org/10.1590/S0037-86821998000400002

ODORIZZI, R.M.F.N. \& GALATI, E.A.B. 2007. Flebotomíneos de várzea do rio Aguapeí, região noroeste do Estado de São Paulo, Brasil. Rev. Saúde Públ. 41(4):645-52.

OLIVEIRA, S.M., AFONSO, R.C.H., DIAS, C.M.G. \& BRAZIL, R.P. 1994. Description of a new species of sand fly Lutzomyia (Pressatia) mamedei n. sp. (Diptera:Psychodidae) from Rio de Janeiro, Brazil. Mem. Inst. Oswaldo Cruz 89:319-320. http://dx.doi.org/10.1590/S007402761994000300006

PAIVA, B.R., PASSOS, L.N., FALQUETO, A., MALAFRONTE, R.S., ANDRADE JUNIOR, H.F. 2004. Single step polymerase chain reaction (PCR) for the diagnosis of the Leishmania (Viannia) subgenus. Rev. Inst. Med. trop. S. Paulo 46(6):335-338.

PIGNATTI, M.G., MAYO, R.C., ALVES, M.J.C.P., SOUZA, S.S.A.L., MACEDO, F. \& PEREIRA, R.M. 1995. Leishmaniose tegumentar americana na região nordeste do Estado de São Paulo, Brasil. Rev. Soc. Bras. Med. Trop. 28(3):243-247.

PINTO, C. 1926. Phlebotomus neivai e Phlebotomus fischeri n. sp.- sobre o aparelho espiracular dos phlebótomos e seu valor específico. Ciência Méd. 4:370-375.

RANDOLPH, S.E. 2000. Ticks and tick-borne disease systems in space and from space. Adv. Parasitol. 47:217-243. http://dx.doi.org/10.1016/ S0065-308X(00)47010-7

RANGEL, E.F. \&. LAINSON R. 2003. Flebotomíneos do Brasil. Editora Fiocruz, Rio de Janeiro.
REINERT, J.F., HARBACH, R.E. \& SALLUM, M.A.M. 2005. Checklist of aedine mosquito espécies (Diptera, Culicidae, Aedini) occurring in Middle and South America (south of the United States) reflecting current generic and subgeneric status. Rev. Bras. Entomol. 49(2):249-252. http://dx.doi. org/10.1590/S0085-56262005000200009

SHIMABUKURO, P.H F., MARASSÁ, A.M. \& GALATI, E.A.B. 2007. Brumptomyia carvalheiroi sp. nov. (Diptera: Psychodidae: Phlebotominae) from Atlantic forest domain, São Paulo State, Brazil. Zootaxa 1637:47-54.

SUPERINTENDÊNCIA DE CONTROLE DE ENDEMIAS - SUCEN. 2005. Encontro de Lutzomyia edwardsi infectada da Grande São Paulo. Rev. Saúde Públ. 39:127-138.

SUPERINTENDÊNCIA DE CONTROLE DE ENDEMIAS / DIVISÃO DE ORIENTAÇÃO TÉCNICA - SUCEN/DOT. 2006. Listagem de espécies de flebotomíneos capturados em focos de transmissão de Leishmaniose Tegumentar Americana (LTA) por município e localidade. Estado de São Paulo - 1985 a 1995. http://www.SUCEN.sp.gov.br/base_dados/ texto_bolva05.htm (último acesso em 24/04/2006). Divisão de Orientação Técnica - DOT/SES-SP.

SUPERINTENDÊNCIA DE CONTROLE DE ENDEMIAS - SUCEN/SP. 2006. http://www.SUCEN.sp.gov.br/doencas/leish_teg/texto_leish_ tegum_pro2.htm. (último acesso em 24/04/2006). Serviços Regionais.

TANIGUCHI, H.H., TOLEZANO, J.E., CÔRREA, F.M.A., MORAES, R.H.P., VEIGA, R.M.O. \& MARASSÁ, A.M. 1990. Epidemiologia da Leishmaniose Tegumentar Americana no Estado de São Paulo, Brasil. I. Composição da fauna flebotomínica no município de São Roque, região de Sorocaba. Rev. Inst. Adolfo Lutz 51(1-2):23-30.

TANIGUCHI, H.H., TOLEZANO, J.E., LAROSA, R., ELIAS, C.R. \& GALATI, E.A.B. 2002. Observações ecológicas de flebotomíneos em área endêmica no município de Eldorado Paulista, Vale do Riberia, estado de São Paulo, Brasil. 1996-1997. Sazonalidade e frequência de Lutzomyia ayrozai em diferentes ecótopos com animais sentinelas em ambiente florestal. Rev. Inst. Adolfo Lutz 6(1):103-112.

TOLEZANO, J.E. 1994. Ecoepidemiological aspects of American cutaneous leishmaniose in the State of São Paulo, Brazil. Mem. Inst.Oswaldo Cruz 83:427-434. http://dx.doi.org/10.1590/S0074-02761994000300026

VASCONCELOS, P.F.C., TRAVASSOS DA ROSA, A.P.A., RODRIGUES, S.G., TRAVASSOS DA ROSA, E.S., DÉGALLIER, N. \& TRAVASSOS DA ROSA, J.F.S. 2001. Inadequate management of natural ecosystem in the Brazilian Amazon region results in the emergence and reemergence of arboviruses. Cad. S. Públ. 17:156-164.

VECTORBASE. http://www.vectorbase.org (último acesso em 09/2010).

WILLIAMS, P. \& CARVALHO, A.L.M. 1979.Description of the female of Lutzomyia (Lutzomyia) dispar, with a redescription of the male (Diptera: Psychodidae: Phlebotominae). J. Med. Entomol. 16(4):325-330.

YOUNG, D.G. \& DUNCAN, M.A. 1994. Guide to the identification and geographic distribution of Lutzomyia sandflies in Mexico, the West Indies, Central and South America (Diptera: Psychodidae). Mem. Am. Entomol. Inst. 54:1-881. 


\section{Apêndices 1 e 2}

Apêndice 1. Lista de espécies de Phlebotominae (Diptera, Psychodidae) do Estado de São Paulo, Brasil, com comentários sobre sua distribuição geográfica. Appendix 1. Checklist of Phlebotominae (Diptera, Psychodidae) from São Paulo state, Brazil, with notes on their geographical distribution.

BRUMPTOMYIINA ARTEMIEV, 1991

Brumptomyia França e Parrot, 1921

avellari (Costa Lima, 1932)

Distribuição: Cássia dos Coqueiros (MZUSP), Corumbataí (Cutolo e Von Zuben 2008), Franca (Martins et al. 1978), Mirandópolis (Odorizzi e Galati 2007), Presidente Venceslau (Forattini 1954), Queiróz (MZUSP), Santa Cruz das Palmeiras (Barretto 1947; Martins et al. 1978; MZUSP), Santo Anastácio (Forattini 1954), Teodoro Sampaio (Martins et al. 1978).

bragai Mangabeira e Sherlock, 1961

Distribuição: Cananéia (Gomes e Galati 1989), Ribeirão Grande (Galati et al. 2010).

brumpti (Larrousse, 1920)

Distribuição: Araraquara (observações não publicadas), Cássia dos Coqueiros (MZUSP), Corumbataí (Cutolo e Von Zuben 2008), Guaíra (Gomes et al. 1978), Ipeúna (Cutolo et al. 2008), Lins [MF] (Larrouse, 1920; Barretto 1947), Martinópolis (Barretto 1947; Martins et al. 1978), Mogi-Guaçu (informações não publicadas), Pereira Barreto (MZUSP), Pirapozinho (Forattini 1954), Pompéia (Barretto 1947; Martins et al. 1978), Presidente Epitácio (Forattini 1954), Presidente Prudente (Barretto 1947; Forattini 1954; Martins et al. 1978), Presidente Venceslau (Forattini 1954), Queiróz (MZUSP), Rancharia (Barretto 1947; Martins et al. 1978), Ribeirão Preto (Barretto 1947; Martins et al. 1978), Santa Cruz das Palmeiras (Barretto 1947; Martins et al. 1978), Santo Anastácio (Forattini 1954), São José dos Campos (Barretto 1947; Martins et al. 1978), São Paulo (Barretto 1947; Martins et al. 1978) Teodoro Sampaio (Martins et al. 1978; Condino et al. 1998).

cardosoi (Barretto e Coutinho, 1941)

Distribuição: Cássia dos Coqueiros (MZUSP), São José dos Campos [M] (Barretto e Coutinho 1941) (Barretto 1947; Martins et al. 1978), São Paulo (observações não publicadas), São Roque (Taniguchi et al. 1990).

carvalheiroi Shimabukuro, Marassá e Galati, 2007

Distribuição: Iporanga, Ribeirão Grande (Shimabukuro et al. 2007; Galati et al. 2010).

cunhai (Mangabeira, 1942)

Distribuição: Cássia dos Coqueiros (MZUSP), Corumbataí (Cutolo e Von Zuben 2008), Iporanga (observações não publicadas), Ribeirão

Grande (Galati et al. 2010), São João da Boa Vista (informações não publicadas), São Sebastião (Brito et al. 2002; Martins et al. 1978).

guimaraesi (Coutinho e Barretto, 1941)

Distribuição: Cássia dos Coqueiros (MZUSP), Corumbataí (Cutolo e Von Zuben 2008), Itaporanga [MF] (Coutinho e Barretto, 1941), Itararé (Barretto 1947; Martins et al. 1978), Una (Ibiúna hoje) (Martins et al. 1978).

mangabeirai (Barretto e Coutinho, 1941)

Distribuição: Campinas (Barretto 1947; Martins et al. 1978), São José dos Campos [M] (Barretto e Coutinho 1941; Martins et al. 1978),

São Paulo (Barretto 1947; Martins et al. 1978), São Roque (Taniguchi et al. 1990).

nitzulescui (Costa Lima, 1932)

Distribuição: Cananéia (Gomes e Galati 1989), Cássia dos Coqueiros (MZUSP), Franca (Martins et al. 1978), Lins [M] (Costa Lima

1932); Martinópolis (Barretto 1947; Martins et al. 1978), Pariquera-Açú, (Gomes e Galati 1987), Presidente Prudente (Barretto 1947;

Martins et al. 1978), Rancharia (Barretto 1947; Martins et al. 1978), São José dos Campos (Barretto 1947; Martins et al. 1978), São Paulo

(Barretto 1947; Martins et al. 1978), São Roque (Taniguchi et al. 1990), Taquarituba (Gomes et al. 1978).

ortizi Martins, Silva e Falcão, 1971

Distribuição: Cássia dos Coqueiros (MZUSP).

pintoi (Costa Lima, 1932)

Distribuição: Araraquara (observações não publicadas); Santa Cruz das Palmeiras (Barretto 1947; Martins et al. 1978); Guaíra

(Gomes et al. 1978).

troglodytes (Lutz, 1922)

Distribuição: Ariri (observações não publicadas), Cananéia (Gomes e Galati 1989), Eldorado (Taniguchi et al. 2002), Iporanga (observações não publicadas), Ribeirão Grande (Galati et al. 2010).

\section{SERGENTOMYIINA ARTEMIEV, 1991}

Micropygomyia Barretto, 1962

(Sauromyia) Artemiev, 1991 
série oswaldoi Barretto, 1962

ferreirana (Barretto, Martins e Pellegrino, 1956)

Distribuição: Apiaí [como L. borgmeieri (sinonímia)] (Martins et al. 1978), Cananéia (Gomes e Galati 1989), Eldorado (Taniguchi et al. 2002), Itirapina (Cutolo et al. 2008), Itupeva [como L. borgmeieri (sinonímia)] (Mayo et al. 1998), Miracatu/Pedro de Toledo (Gomes et al. 1990b), Pariquera-Açú (Gomes e Galati 1987), São Sebastião (Brito et al. 2002) [como L. borgmeieri (sinonímia)].

longipennis (Barretto, 1946)

Distribuição: Cássia dos Coqueiros (MZUSP), Guaíra (Gomes et al 1978; MZUSP), Luís Antônio (Forattini et al. 1976a).

petari Galati,Marassá e Andrade, 2003

Distribuição: Iporanga [MF] (Galati et al. 2003), Ribeirão Grande (Galati et al. 2003; Galati et al. 2010).

quinquefer (Dyar, 1929)

Distribuição: registrada no estado de São Paulo (Casanova com. pess.)

(Micropygomyia) s. str. Barretto, 1962

série cayennensis Fairchild, 1955

schreiberi (Martins, Falcão e Silva, 1975)

Distribuição: Caraguatatuba (Martins et al. 1978), São Sebastião (Martins et al. 1978; Brito et al. 2002).

LUTZOMYIINA ABONNENC E LÈGER, 1976

Sciopemyia Barretto, 1962

microps (Mangabeira, 1942)

Distribuição: Cananéia (Gomes e Galati 1989), Iporanga (observações não publicadas), Miracatu/Pedro de Toledo (Gomes et al. 1990b),

Ribeirão Grande (Galati et al. 2010), São Sebastião (Martins et al. 1978).

sordellii (Shannon e Del Ponte, 1927)

Distribuição: Ipeúna (Cutolo et al. 2008), São Sebastião (Brito et al. 2002), Ribeirão Grande (Galati et al. 2010).

Lutzomyia França, 1924

(Castromyia) Mangabeira, 1942

amarali (Barretto e Coutinho, 1940)

Distribuição: Cananéia (Gomes e Galati 1989), Iporanga (observações não publicadas), Pariquera-Açú (Gomes e Galati 1987),

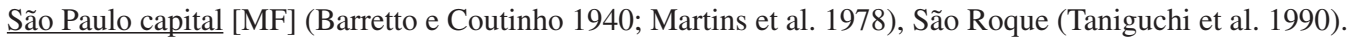

castroi (Barretto e Coutinho, 1941)

Distribuição: Itaporanga [M] (Barretto e Coutinho, 1941; Barretto 1947).

(Lutzomyia) s. str.

almerioi Galati e Nunes, 1999

Distribuição: registrada no Estado de São Paulo (observações não publicadas).

dispar Martins e Silva, 1963

Distribuição: Cajurú (Martins et al. 1978; Williams e Carvalho 1979), Cássia dos Coqueiros (Martins et al. 1978; Williams e Carvalho 1979).

longipalpis (Lutz e Neiva, 1912)

Distribuição: Andradina (SUCEN/DOT 2006), Araçatuba (SUCEN/DOT 2006), Auriflama (SUCEN/DOT 2006), Avanhandava (SUCEN/ DOT 2006), Bento de Abreu (SUCEN/DOT 2006), Birigui (SUCEN/DOT 2006), Cássia dos Coqueiros (Forattini et al. 1976a; Martins et al. 1978), Castilho (SUCEN/DOT 2006), Coroados (SUCEN/DOT 2006), Espírito Santo do Pinhal (Costa et al. 1997), Glicério (SUCEN/ DOT 2006), Guaraçaí (SUCEN/DOT 2006), Guararapes (SUCEN/DOT 2006), Iporanga (observações não publicadas), Itapura (SUCEN/ DOT 2006), Itirapina (Cutolo et al. 2008), Itupeva (Pignatti et al. 1995; Costa et al. 1997; Mayo et al. 1998), Lavínia (SUCEN/DOT 2006), Mirandópolis (SUCEN/DOT 2006), Muritinga do Sul (SUCEN/DOT 2006), Penápolis (SUCEN/DOT 2006), Pereira Barreto (SUCEN/ DOT 2006), Pirapora do Bom Jesus (Forattini et al. 1976a; Martins et al. 1978; SUCEN/DOT 2006), Promissão (SUCEN/DOT 2006), Rubiácea (SUCEN/DOT 2006), Salto do Pirapora (Forattini et al. 1970; Martins et al. 1978), Santo Antônio do Aracanguá (SUCEN/DOT 2006), Socorro (Costa et al. 1997), Valparaíso (SUCEN/DOT 2006). 
(Migonemyia) s. str.

migonei (França, 1920)

Distribuição: Adamantina (SUCEN/SP 2006), Andradina (Forattini 1954; Martins et al. 1978), Araçariguama (SUCEN/SP 2006), Araçatuba (Martins et al. 1978), Araçoiaba da Serra (SUCEN/SP 2006), Araraquara (Martins et al. 1978), Assis (SUCEN/SP 2006), Avanhandava (Martins et al. 1978), Avaré (Martins et al. 1978), Barra Bonita (Martins et al. 1978), Barra do Turvo (SUCEN/SP 2006), Birigui (Martins et al. 1978), Boituva (SUCEN/SP 2006), Caçapava (SUCEN/SP 2006), Cachoeira Paulista (SUCEN/SP 2006), Cajati (SUCEN/SP 2006), Cajamar (SUCEN/SP 2006), Cananéia (Gomes e Galati, 1989), Capão Bonito (Martins et al. 1978), Catanduva (Martins et al. 1978), Cerquilho (SUCEN/SP 2006), Cotia (SUCEN/SP 2006), Dracena (Forattini 1954), Eldorado (Taniguchi et al. 2002), Embu das Artes (SUCEN 2005), Franca (Martins et al. 1978), Franco da Rocha (SUCEN/SP 2006), Gracinópolis (Forattini 1954), Guareí (SUCEN/SP 2006), Ibira (SUCEN/SP 2006), Iguape (Galati e Gomes 1992), Ilhabela (SUCEN/SP 2006), Inúbia Paulista (SUCEN/SP 2006), Iperó (SUCEN/SP 2006), Iporanga (observações não publicadas; SUCEN/SP 2006), Itaóca (SUCEN/SP 2006), Itapirapuã Paulista (SUCEN/SP 2006), Itaporanga (Martins et al. 1978), Itariri (SUCEN/SP 2006), Itirapina (Cutolo et al. 2008), Itu (SUCEN/SP 2006), Itupeva (Mayo et al. 1998; Pignatti et al. 1995), Jacupiranga (SUCEN/SP 2006), José Bonifácio (Martins et al. 1978), Junqueirópolis (Forattini 1954; Martins et al. 1978), Juquiá (SUCEN/SP 2006), Lagoinha (SUCEN/SP 2006), Leme (Martins et al. 1978), Lins (Martins et al. 1978), Mairiporã (SUCEN/SP 2006), Marília (Martins et al. 1978), Martinópolis (Martins et al. 1978), Miracatu/Pedro de Toledo (Gomes et al. 1990b), Mogi das Cruzes (Martins et al. 1978; SUCEN/SP 2006), Mogi Mirim (Martins et al. 1978), Monte Aprazível (Martins et al. 1978), Natividade da Serra (SUCEN/SP 2006), Novo Horizonte (Martins et al. 1978), Olímpia (Martins et al. 1978), Osasco (SUCEN/ SP 2006), Osvaldo Cruz (SUCEN/SP 2006), Ourinhos (Martins et al. 1978), Pacaembu (Barretto 1947; Forattini 1954; Martins et al. 1978), Palmital (Martins et al. 1978), Pariquera-Açú (Gomes et al. 1978; Gomes et a1. 1986; Gomes e Galati 1987; SUCEN/SP 2006), Pedro de Toledo (Forattini et al. 1976b; Domingos et al. 1998, SUCEN/SP 2006), Penápolis (Martins et al. 1978; Mayo et al. 1998), Piedade (Martins et al. 1978; SUCEN/SP 2006), Piraju (Martins et al. 1978; SUCEN/SP 2006), Pirapora do Bom Jesus (SUCEN/SP 2006), Pirapozinho (Forattini 1954), Platina (SUCEN/SP 2006), Pompéia (Martins et al. 1978), Porangaba (SUCEN/SP 2006), Porto Feliz (Martins et al. 1978; SUCEN/SP 2006), Presidente Epitácio (Barretto 1947; Forattini 1954; Martins et al. 1978), Presidente Prudente (Forattini 1954; Barretto 1947; Martins et al. 1978), Presidente Venceslau (Barretto 1947; Forattini 1954; Martins et al. 1978), Queiróz (MZUSP), Rancharia (Martins et al. 1978), Regente Feijó (Martins et al. 1978), Registro (SUCEN/SP 2006), Ribeira (SUCEN/SP 2006), Ribeirão Grande (Galati et al. 2010), Salto (SUCEN/SP 2006), Salto Grande (SUCEN/SP 2006), Santa Cruz do Rio Pardo (SUCEN/ SP 2006), Santo Anastácio (Barretto 1947; Forattini 1954; Martins et al. 1978), Santo Antônio do Jardim (SUCEN/SP 2006), São Luís do Paraitinga (SUCEN/SP 2006), São Pedro do Turvo (SUCEN/SP 2006), São José do Rio Preto (Barretto 1947; Martins et al. 1978; SUCEN/SP 2006), São José dos Campos (Barretto 1947; Martins et al. 1978; SUCEN/SP 2006), São Paulo (Barretto 1947; Martins et al. 1978; SUCEN/SP 2006; FSP), São Roque (Taniguchi et al. 1990; SUCEN/SP 2006), São Sebastião (Brito et al. 2002; SUCEN/SP 2006), São Sebastião da Grama (SUCEN/SP 2006), Sarutaiá (SUCEN/SP 2006), Silveiras (SUCEN/SP 2006), Sorocaba (Barretto 1947; Martins et al. 1978; SUCEN/SP 2006), Tamoio (Barretto 1947; Martins et al. 1978), Tanabi (Barretto 1947; Martins et al. 1978), Tatuí (SUCEN/SP 2006), Tejupa (SUCEN/SP 2006), Teodoro Sampaio (Condino et al. 1998; SUCEN/SP 2006), Tietê (Martins et al. 1978), Tupã (Martins et al. 1978), Ubatuba (SUCEN/SP 2006), Una (Ibiúna hoje) (Martins et al. 1978), Valparaíso (Barretto 1947; Forattini 1954, Martins et al. 1978), Vera Cruz (Martins et al. 1978).

rabelloi (Galati e Gomes 1992)

Distribuição: Cananéia (Galati e Gomes 1992), Iguape [MF], Iporanga (Galati e Gomes 1992), Pedro de Toledo, (Galati e Gomes 1992), Ribeirão Grande (Galati et al. 2010).

vaniae Galati, Fonseca e Marassá, 2007

Distribuição: Iporanga [MF] (Galati, Fonseca e Marassá, 2007)

(Blancasmyia) Galati, 1995

bursiformis (Floch e Abonnenc, 1944)

Distribuição: Guaíra [como L. baityi (sinonímia)] (Gomes et al. 1978)

Pintomyia Costa Lima, 1932

(Pintomyia) s. str.

bianchigalatiae (Andrade-Filho, Aguiar, Dias e Falcão, 1999)

Distribuição: Corumbataí (Cutolo e Von Zuben 2008).

christenseni (Young e Duncan, 1994)

Distribuição: registrada no Estado de São Paulo (observações não publicadas)

fischeri (Pinto, 1926)

Distribuição: Alto da Serra (Martins et al. 1978), Alumínio (SUCEN/SP 2006), Amparo (Martins et al. 1978), Apiaí (Martins et al. 1978), Araçariguama (SUCEN/SP 2006), Araçoiaba da Serra (SUCEN/SP 2006), Arandu (SUCEN/SP 2006), Assis (SUCEN/SP 2006), Avaré (Martins et al. 1978), Barra do Turvo (SUCEN/SP 2006), Bananal (SUCEN/SP 2006), Bateia (Martins et al. 1978), Boituva (SUCEN/SP 2006), Cabreúva (Martins et al. 1978), Cachoeira Paulista (SUCEN/SP 2006), Cajamar (SUCEN/SP 2006), Cajati (SUCEN/SP 2006), Cajuru (Martins et al. 1978), Campinas (Martins et al. 1978; Gomes et al. 1989), Campos do Jordão (Martins et al. 1978), Cananéia (Gomes 
e Galati, 1989), Capão Bonito (Martins et al. 1978), Capela do Alto (SUCEN/SP 2006), Caraguatatuba (Martins et al. 1978; SUCEN/SP 2006), Catanduva (Martins et al. 1978), Cerquilho (SUCEN/SP 2006), Cotia (SUCEN/SP 2006), Cubatão (Martins et al. 1978), Cunha (SUCEN/SP 2006), Dourado (Gomes et al. 1989), Eldorado (SUCEN/SP 2006; Taniguchi et al. 2002), Embu das Artes (SUCEN 2005), Embu-Guaçu (SUCEN/SP 2006), Franca (Martins et al. 1978), Francisco Morato (SUCEN/SP 2006), Guaratinguetá (Martins et al. 1978), Guarujá (Martins et al. 1978), Ibiúna (SUCEN/SP 2006), Igarapava (Martins et al. 1978), Iguape (Martins et al. 1978), Igaratá (SUCEN/SP 2006), Ilhabela (SUCEN/SP 2006), Inúbia Paulista (SUCEN/SP 2006), Iperó (SUCEN/SP 2006), Iporanga (obervações não publicadas; SUCEN/SP 2006), Itabera (SUCEN/SP 2006), Itanhaém (Martins et al. 1978), Itaóca (SUCEN/SP 2006), Itapecerica da Serra (Martins et al. 1978), Itapetininga (SUCEN/SP 2006), Itaporanga (Martins et al. 1978; Gomes et al. 1989), Itariri (SUCEN/SP 2006), Itu (SUCEN/SP 2006), Itupeva (Mayo et al. 1998), Jacareí (Martins et al. 1978), Jacupiranga (Martins et al. 1978; SUCEN/SP 2006), Jambeiro (SUCEN/ SP 2006), Jundiaí (Martins et al. 1978), Junqueirópolis (Forattini 1954), Juquiá (SUCEN/SP 2006), Lagoinha (SUCEN/SP 2006), Leme (Martins et al. 1978), Mairinque (SUCEN/SP 2006), Mairiporã (SUCEN/SP 2006), Miracatu/Pedro de Toledo (Gomes et al. 1990b), Mogi das Cruzes (Martins et al. 1978; SUCEN/SP 2006), Mogi Mirim (Martins et al. 1978), Monte Aprazível (Martins et al. 1978), Natividade da Serra (SUCEN/SP 2006), Novo Horizonte (Martins et al. 1978), Olímpia (Martins et al. 1978), Osasco (Martins et al. 1978), Pacaembu (Forattini 1954), Pariquera-Açú (Gomes et al. 1978; Gomes et al. 1986; Gomes e Galati 1987; SUCEN/SP 2006), Platina (SUCEN/SP 2006), Pedro de Toledo (Forattini et al. 1976b; Domingos et al. 1998; SUCEN/SP 2006), Piedade (Martins et al. 1978; SUCEN/SP 2006), Pindamonhangaba (Martins et al. 1978), Piracicaba (Martins et al. 1978), Piraju (SUCEN/SP 2006), Pirapora do Bom Jesus (SUCEN/ SP 2006), Pirapozinho (Forattini 1954), Pompéia (Martins et al. 1978), Porangaba (SUCEN/SP 2006), Porto Feliz (Martins et al. 1978; SUCEN/SP 2006), Prainha (Martins et al. 1978), Presidente Epitácio (Forattini 1954), Presidente Prudente (Forattini 1954; Martins et al. 1978; SUCEN/SP 2006), Presidente Venceslau (Forattini 1954), Rancharia (Martins et al. 1978), Registro (SUCEN/SP 2006), Ribeira (SUCEN/SP 2006), Ribeirão Grande (Galati et al. 2010), Ribeirão Preto (Martins et al. 1978), Rocinha (Martins et al. 1978), Salesópolis (Martins et al. 1978), Salto (SUCEN/SP 2006), Salto do Pirapora (SUCEN/SP 2006), Salto Grande (SUCEN/SP 2006), Santa Branca (SUCEN/SP 2006), Santa Cruz das Palmeiras (Martins et al. 1978), Santa Cruz do Rio Pardo (SUCEN/SP 2006), Santo Anastácio (Forattini 1954), Santo Antônio do Jardim (SUCEN/SP 2006), São Sebastião (Martins et al. 1978; Brito et al. 2002; SUCEN/SP 2006), São José dos Campos (Martins et al. 1978), São José do Rio Pardo (Martins et al. 1978), São Luís do Paraitinga (SUCEN/SP 2006), São Paulo [M] (Pinto, 1926)(Martins et al. 1978), São Pedro do Turvo (SUCEN/SP 2006), São Roque (SUCEN/SP 2006; Taniguchi et al. 1990), São Vicente (Martins et al. 1978), Sarutaiá (SUCEN/SP 2006), Sorocaba (Martins et al. 1978; SUCEN/SP 2006), Tamoio (Martins et al. 1978), Taubaté (SUCEN/SP 2006), Tejupa (SUCEN/SP 2006), Tietê (Martins et al. 1978; SUCEN/SP 2006), Timburi (SUCEN/SP 2006), Ubatuba (SUCEN/SP 2006), Una (Ibiúna hoje) (Martins et al. 1978), Vera Cruz (Martins et al. 1978).

pessoai (Coutinho e Barretto, 1940)

Distribuição: Alfredo de Castilho (Barretto 1947), Assis (SUCEN/SP 2006), Avanhandava (Martins et al. 1978), Andradina (Forattini 1954; Martins et al. 1978), Araçatuba (Barretto 1947; Martins et al. 1978), Araraquara (Martins et al. 1978), Assis (Martins et al. 1978), Avaré (Martins et al. 1978), Barra Bonita (Martins et al. 1978), Birigui (Martins et al. 1978), Boituva (SUCEN/SP 2006), Capela do Alto (SUCEN/SP 2006), Catanduva (Martins et al. 1978), Chavantes (SUCEN/SP 2006), Corumbataí (Cutolo e Von Zuben 2008), Dracena (Forattini 1954), Gália (SUCEN/SP 2006), Gracinópolis (Forattini 1954), Igaratá (SUCEN/SP 2006), Inúbia Paulista (SUCEN/SP 2006), Ipeúna (Cutolo et al. 2008), Itupeva (Mayo et al. 1998), Itu (SUCEN/SP 2006), Jambeiro (SUCEN/SP 2006), José Bonifácio (Martins et al. 1978), Junqueirópolis (Forattini 1954), Lins (Martins et al. 1978), Luís Antônio (Forattini et al. 1976b; Martins et al. 1978), Marília (Barretto 1947; Martins et al. 1978), Martinópolis (Barretto 1947; Martins et al. 1978; SUCEN/SP 2006), Mirandópolis (SUCEN/SP 2006), Mirassol (Barretto 1947), Monte Aprazível (Barretto 1947; Martins et al. 1978), Novo Horizonte (Barretto 1947; Martins et al. 1978), Olímpia (Barretto 1947; Martins et al. 1978), Osvaldo Cruz (SUCEN/SP 2006), Ourinhos (Barretto 1947; Martins et al. 1978), Pacaembu (Forattini 1954), Palmital (Barretto 1947; Martins et al. 1978), Penápolis (Barretto 1947; Martins et al. 1978), Pindorama (Barretto 1947; Martins et al. 1978), Piraju (SUCEN/SP 2006), Pirajuí (Barretto 1947; Martins et al. 1978), Pirapora do Bom Jesus (SUCEN/SP 2006), Pirapozinho (Forattini 1954), Pompéia [MF] (Barretto 1947; Martins et al. 1978), Porangaba (SUCEN/SP 2006), Porto Feliz (SUCEN/ SP 2006), Presidente Alves (Barretto 1947), Presidente Epitácio (Barretto 1947; Forattini 1954; Martins et al. 1978), Presidente Prudente (Barretto 1947; Forattini 1954; Martins et al. 1978), Presidente Venceslau (Barretto 1947; Forattini 1954; Martins et al. 1978), Promissão (Barretto 1947; Martins et al. 1978), Rancharia (Barretto 1947; Martins et al. 1978), Regente Feijó (Barretto 1947; Martins et al. 1978), Registro (SUCEN/SP 2006), Salto (SUCEN/SP 2006), Salto Grande (SUCEN/SP 2006), Santa Cruz do Rio Pardo (SUCEN/SP 2006), Santo Anastácio (Forattini 1954; Martins et al. 1978), Santo Antônio do Jardim (SUCEN/SP 2006), São João da Boa Vista (SUCEN/ SP 2006), São José do Rio Preto (Barretto 1947; Martins et al. 1978), São Pedro do Turvo (SUCEN/SP 2006), Tamoio (Barretto 1947; Martins et al. 1978), Teodoro Sampaio (Condino et al. 1998; SUCEN/SP 2006), Tejupa (SUCEN/SP 2006), Timburi (SUCEN/SP 2006), Tupã (Martins et al. 1978), Valparaíso (Forattini 1954; Martins et al. 1978), Vera Cruz (Martins et al. 1978).

(Pifanomyia) Ortiz e Scorza, 1963

série monticola Artemiev, 1991

misionensis (Castro, 1959)

Distribuição: Cachoeira Paulista (SUCEN/SP 2006), Cunha (SUCEN/SP 2006), Iporanga (observações não publicadas), Lagoinha (SUCEN/SP 2006), Piraju (SUCEN/SP 2006), Pirapora do Bom Jesus (SUCEN/SP 2006), São José dos Campos (SUCEN/SP 2006), São Roque (Taniguchi et al. 1990), Sarutaiá (SUCEN/SP 2006), Silveiras (SUCEN/SP 2006).

monticola (Costa Lima, 1932)

Distribuição: Araçoiaba da Serra (SUCEN/SP 2006), Bananal? (Martins et al. 1978; SUCEN/SP 2006), Bofete (SUCEN/SP 2006), Cachoeira Paulista (SUCEN/SP 2006), Cananéia (Gomes e Galati 1989), Cajamar (SUCEN/SP 2006), Capela do Alto (SUCEN/SP 2006), 
Cerquilho (SUCEN/SP 2006), Corumbataí (Cutolo e Von Zuben 2008), Cotia (SUCEN/SP 2006), Eldorado (Taniguchi et al. 2002), Embu das Artes (SUCEN 2005), Gália (SUCEN/SP 2006), Ibiúna (SUCEN/SP 2006), Iperó (SUCEN/SP 2006), Ipeúna (Cutolo et al. 2008), Iporanga (SUCEN/SP 2006), Itaporanga (Barretto 1947; Martins et al. 1978), Itupeva (Mayo et al. 1998), Itu (SUCEN/SP 2006), Jundiaí (Barretto 1947; Martins et al. 1978), Mairinque (SUCEN/SP 2006), Mogi das Cruzes (Barretto 1947; Martins et al. 1978; MZUSP), Pilar do Sul (SUCEN/SP 2006), Pindamonhagaba (Barretto 1947; Martins et al. 1978; MZUSP), Pirapora do Bom Jesus (SUCEN/SP 2006), Porto Feliz (SUCEN/SP 2006), Presidente Venceslau (Forattini 1954), Ribeirão Grande (Galati et al. 2010), Ribeirão Preto (Barretto 1947; Martins et al. 1978), Rocinha (Barretto 1947; Martins et al. 1978), Salesópolis (Barretto 1947; Martins et al. 1978; MZUSP), Santa Cruz das Palmeiras (Barretto 1947; Martins et al. 1978), Santo Anastácio (Forattini 1954), Santo Antônio do Jardim (SUCEN/SP 2006), São José dos Campos (Martins et al. 1978), São José do Rio Pardo (Martins et al. 1978), São Paulo [M] (Costa Lima 1932) (Barretto 1947; Martins et al. 1978), São Roque (Taniguchi et al. 1990; SUCEN/SP 2006), Sorocaba (SUCEN/SP 2006), Tatuí (SUCEN/SP 2006).

Expapillata Galati,1995

firmatoi (Barretto, Martins e Pellegrino, 1956)

Distribuição: Araçoiaba da Serra (SUCEN/SP 2006), Barra do Turvo (SUCEN/SP 2006), Boituva (SUCEN/SP 2006), Capão Bonito (Martins et al. 1978), Cássia dos Coqueiros (Forattini et al. 1976a), Cerquilho (SUCEN/SP 2006), Eldorado (Taniguchi et al. 2002), Gália (SUCEN/SP 2006), Ilhabela (SUCEN/SP 2006), Itaóca (SUCEN/SP 2006), Iporanga (observações não publicadas), Itaporanga (SUCEN/SP 2006), Itupeva (Pignatti et al. 1995; Mayo et al. 1998), Luís Antônio (Forattini et al. 1976a,b), Mairiporã (SUCEN/SP 2006), Platina (SUCEN/SP 2006), Pedro de Toledo (Forattini et al. 1976a,b; Domingos et al. 1998; SUCEN/SP 2006), Piraju (SUCEN/SP 2006), Porangaba (SUCEN/SP 2006), Porto Feliz (SUCEN/SP 2006), Ribeirão Grande (Galati et al. 2010), Santo Antônio do Jardim (SUCEN/ SP 2006), São Pedro do Turvo (SUCEN/SP 2006), São Roque (Taniguchi et al. 1999), São Sebastião (Martins et al. 1978; Brito et al. 2002) [F], Taquarituba (Forattini et al. 1976a) Tatuí (SUCEN/SP 2006), Taubaté (SUCEN/SP 2006), Teodoro Sampaio (Condino et al. 1998; SUCEN/SP 2006), Ubatuba (SUCEN/SP 2006).

Pressatia Mangabeira, 1942

choti (Floch e Abonnenc, 1941)

Distribuição: Luís Antônio (Forattini et al. 1976b) - possível erro de identificação, as fêmeas são muito parecidas com as fêmeas de

Pintomyia (Pintomyia) christenseni (Young \& Duncan, 1994) e de P. (P.) mamedei (Oliveira et al. 1994).

trispinosa (Mangabeira, 1942)

Distribuição: Igarapava (Barretto 1943) - possível erro de identificação, fêmeas são similares às fêmeas do gênero Pintomyia (Pintomyia) sp.

Evandromyia Mangabeira, 1941

(Aldamyia) Galati, 1995

carmelinoi (Ryan, Fraiha, Lainson e Shaw, 1986)

Distribuição: Guaíra (como Ev. lenti, Forattini et al. 1976a).

lenti (Mangabeira, 1938)

Distribuição: Cachoeira Paulista (SUCEN/SP 2006), Cássia dos Coqueiros (MZUSP), Ipeúna (Cutolo et al. 2008); Itupeva (Mayo et al. 1998), Pacaembu (Forattini et al. 1976a), Pirapora do Bom Jesus (SUCEN/SP 2006).

termitophila (Martins, Falcão e Silva, 1964)

Distribuição: Cássia dos Coqueiros (Galati e Gomes 1977, descrição da fêmea), Corumbataí (Cutolo e Von Zuben 2008); Guaíra (Galati e Gomes 1977, descrição da fêmea); São Roque (Taniguchi et al. 1990).

(Evandromyia) s. str.

série infraspinosa Fairchild, 1955

bourrouli (Barretto e Coutinho, 1941)

Distribuição: Santa Cruz das Palmeiras [MF] (Barretto e Coutinho, 1941)

série rupicola Young e Fairchild, 1974

correalimai (Martins, Coutinho e Luz, 1970)

Distribuição: Ribeirão Grande (Galati et al. 2010); Taquarituba (Forattini et al. 1976a; Martins et al. 1978).

rupicola (Martins, Godoy e Silva, 1962)

Distribuição: registrada no Estado de São Paulo (observações não publicadas)

(Barrettomyia) Martins e Silva, 1968

série tupynambai Artemiev, 1991 
petropolitana (Martins e Silva, 1968)

Distribuição: Cananéia (Gomes e Galati 1989), Ilhabela (SUCEN/SP 2006), Pariquera-Açú (Gomes e Galati 1987), Ubatuba (SUCEN/ SP 2006).

série cortelezzii Galati, 1995

cortelezzii (Brèthes, 1923)

Distribuição: Araçatuba (Barretto 1947), Dourado (Galati et al. 1989), Guaíra (Gomes et al. 1978; Galati et al. 1989), Ipeúna (Cutolo et al. 2008), Itupeva (Mayo et al. 1998), Mogi das Cruzes (Barretto 1947), Pariquera-Açú (Gomes et al. 1978), Piraju (SUCEN/SP 2006), Porangaba (SUCEN/SP 2006), Porto Feliz (SUCEN/SP 2006), São Paulo (Barretto 1947), Salto Grande (SUCEN/SP 2006), Taquarituba (Gomes et al. 1978).

edwardsi (Mangabeira, 1941)

Distribuição: Cananéia (Gomes e Galati 1989), Cotia (SUCEN 2005), Eldorado (Taniguchi et al. 2002), Embu das Artes (SUCEN 2005), Ilhabela (SUCEN/SP 2006), Miracatu/Pedro de Toledo (Gomes et al. 1990b), Pariquera-Açú (Gomes et al. 1978; Gomes e Galati 1987), Pirapora do Bom Jesus (SUCEN/SP 2006), Ribeirão Grande (Galati et al. 2010), São José dos Campos (MZUSP); São Paulo (SUCEN 2005), São Roque (Taniguchi et al. 1990), São Sebastião (Brito et al. 2002), Ubatuba (SUCEN/SP 2006).

sallesi (Galvão e Coutinho, 1939)

Distribuição: Araçatuba [MF] (Galvão e Coutinho, 1939; Martins et al. 1978); Cássia dos Coqueiros (Galati et al. 1989), Itirapina (Cutolo et al. 2008); Mogi das Cruzes (Martins et al. 1978), Mogi-Guaçú (Galati et al. 1989), São Paulo (Martins et al. 1978).

PSYCHODOPYGINA GALATI, 1995

Psathyromyia Barretto, 1962

(Forattiniella) Vargas, 1978

aragaoi (Costa Lima, 1932)

Distribuição: Cássia dos Coqueiros (MZUSP), Corumbataí (Cutolo e Von Zuben 2008), Igarapava (Barretto 1947).

brasiliensis (Costa Lima, 1932)

Distribuição: Cajuru (Barretto 1947; Martins et al. 1978), Cássia dos Coqueiros (Barretto 1947); Igarapava (Barretto 1947; Martins et al. 1978; MZUSP).

lutziana (Costa Lima, 1932)

Distribuição: Guaíra (Gomes et al. 1978), Igarapava (Barretto 1947; Martins et al. 1978).

pascalei (Coutinho e Barretto, 1940)

Distribuição: Cananéia (Gomes e Galati 1989), Cajamar (SUCEN/SP 2006), Iporanga (observações não publicadas), Miracatu (SUCEN/ SP 2006), Natividade da Serra (SUCEN/SP 2006), Miracatu/Pedro de Toledo (Gomes et al. 1990b), Pariquera-Açú (Gomes e Galati 1987), Pedro de Toledo (Domingos et al. 1998; SUCEN/SP 2006), Pirapora do Bom Jesus (SUCEN/SP 2006), Ribeirão Grande (Galati et al. 2010), Salesópolis (Barretto 1947; Martins et al. 1978), São José dos Campos (Barretto 1947; Martins et al. 1978), São Paulo [M] (Coutinho e Barretto, 1940; Barretto 1947; Martins et al. 1978), São Sebastião (Brito et al. 2002; SUCEN/SP 2006), São Roque (Taniguchi et al. 1990), Ubatuba (SUCEN/SP 2006).

(Xiphomyia) Artemiev, 1991

hermanlenti (Martins, Silva e Falcão, 1970)

Distribuição: Mirandópolis (Odorizzi e Galati, 2007)

(Psathyromyia) s.str.

série lanei Theodor, 1965

lanei (Barretto e Coutinho, 1941)

Distribuição: Apiaí (Martins et al. 1978), Bananal (Martins et al. 1978), Cananéia (Gomes e Galati 1989, Gomes et al. 1990a), Caraguatatuba (Martins et al. 1978), Casa Grande [MF] (Salesópolis hoje), Ilhabela (SUCEN/SP 2006), Iporanga (observações não publicadas), Itaporanga (Baretto 1947; Martins et al. 1978), Miracatu/Pedro de Toledo (Gomes et al. 1990b), Pariquera-Açú (Gomes e Galati 1987), Ribeirão Grande (Galati et al. 2010), São Sebastião (Brito et al. 2002* identificada como série lanei), Ubatuba (SUCEN/SP 2006).

pelloni (Sherlock e Alencar, 1959)

Distribuição: Apiaí (Martins et al. 1978), Caraguatatuba (Martins et al. 1978), São Sebastião (Brito et al. 2002; Martins et al. 1978).

série shannoni Fairchild, 1955

pestanai (Barretto e Coutinho, 1941) 
Distribuição: Casa Grande [F] (Salesópolis hoje), Francisco Morato (SUCEN/SP 2006), Mairiporã (SUCEN/SP 2006), Mogi das Cruzes (SUCEN/SP 2006), Pedro de Toledo (Domingos et al. 1998; SUCEN/SP 2006), Piedade (Barretto 1947; SUCEN/SP 2006), Presidente Venceslau (Forattini 1954; Martins et al. 1978), Queiróz (MZUSP), Salesópolis (Barretto 1947; Martins et al. 1978; SUCEN/SP 2006), Santa Isabel (SUCEN/SP 2006), Santo Anastácio (Forattini 1954; Martins et al. 1978), São Paulo [M] (Barretto e Coutinho, 1941)(Barreto 1947; Martins et al. 1978).

punctigeniculata (Floch e Abonnenc, 1944)

Distribuição: Guaíra (Gomes et al. 1978).

shannoni (Dyar, 1929)

Distribuição: Andradina (Barretto 1947; Forattini 1954; Martins et al. 1978), Araçatuba (Barretto 1947; Martins et al. 1978), Birigui (Barretto 1947; Martins et al. 1978), Buri (SUCEN/SP 2006), Campinas (Barretto 1947; Martins et al. 1978), Cananéia (Gomes e Galati 1989), Dracena (Forattini 1954), Eldorado (Taniguchi et al. 2002), Gracinópolis (Forattini 1954), Inúbia Paulista (SUCEN/SP 2006), Itaporanga (Barretto 1947; Martins et al. 1978; MZUSP), Itupeva (Mayo et al. 1998), Junqueirópolis (Forattini 1954; Martins et al. 1978), Martinópolis (Barretto 1947; Martins et al. 1978; MZUSP), Mogi das Cruzes (Barretto 1947; Martins et al. 1978; MZUSP), Olímpia (Barretto 1947; Martins et al. 1978), Pacaembu (Forattini 1954; Martins et al. 1978), Paulo de Faria (SUCEN/SP 2006), Piedade (Barretto 1947; Martins et al. 1978), Pilar do Sul (SUCEN/SP 2006), Piraju (SUCEN/SP 2006), Pirapozinho (Forattini 1954), Pompéia (Barretto 1947; Martins et al. 1978), Pontes Gestal (SUCEN 2006), Porangaba (SUCEN 2006), Presidente Alves (Barretto 1947; Martins et al. 1978), Presidente Epitácio (Forattini 1954), Presidente Prudente (Barretto 1947; Forattini 1954; Martins et al. 1978), Presidente Venceslau, (Forattini 1954; Martins et al. 1978), Regente Feijó (Barretto 1947; Martins et al. 1978), Salesópolis (Barretto 1947; Martins et al. 1978), Santo Anastácio, (Barretto 1947; Forattini 1954; Martins et al. 1978), São José do Rio Pardo (Barretto 1947; Martins et al. 1978), São José dos Campos (Barretto 1947; Martins et al. 1978; MZUSP), São Paulo (Barretto 1947; Martins et al. 1978), São Roque (Taniguchi et al. 1990), São Sebastião (SUCEN/SP 2006), Teodoro Sampaio (Condino et al. 1998; SUCEN/SP 2006), Valparaíso (Barretto 1947; Forattini 1954; Martins et al. 1978), Vera Cruz (Barretto 1947; Martins et al. 1978).

Martinsmyia Galati, 1995

grupo alphabetica Fairchild, 1955

alphabetica (Fonseca, 1936)

Distribuição: Alumínio (SUCEN/SP 2006), Cajamar (SUCEN/SP 2006), Chavantes (SUCEN/SP 2006); Cotia (SUCEN/SP 2006), Embu-Guaçu (SUCEN/SP 2006), Juqueri (Barretto 1947; Martins et al. 1978), Itupeva (Mayo et al. 1998), Mairiporã (SUCEN/SP 2006, Martins et al. 1978), Mogi das Cruzes (Barretto 1947; Martins et al. 1978; SUCEN/SP 2006), Piedade (SUCEN/SP 2006), Piraju (SUCEN/ SP 2006), Pirapora do Bom Jesus (SUCEN/SP 2006), São Paulo [MF] (Fonseca, 1936) (Barretto 1947; FSP; Martins et al. 1978), Salto do Pirapora (SUCEN/SP 2006), São Roque (SUCEN/SP 2006; Taniguchi et al. 1990).

Bichromomyia Artemiev, 1991

flaviscutellata (Mangabeira, 1942)

Distribuição: Cananéia [Gomes e Galati 1989, Gomes et al. 1990a), Pariquera-Açú (Gomes e Galati 1987).

Psychodopygus Mangabeira, 1941

série arthuri Barretto, 1962

arthuri (Fonseca, 1936)

Distribuição: Cajamar (SUCEN/SP 2006), Campinas, (Barretto 1947; Martins et al. 1978), Caraguatatuba (SUCEN/SP 2006), Ferraz de Vasconcelos (FSP/USP), Jacareí (Barretto 1947; Martins et al. 1978), Mogi das Cruzes (Barretto 1947; Martins et al. 1978), Natividade da Serra (SUCEN/SP 2006), Osasco (Barretto 1947; Martins et al. 1978, Galati 1981), Piedade (Barretto 1947; Martins et al. 1978), Pirapora do Bom Jesus (SUCEN/SP 2006), Poá (Galati 1981), Porto Feliz (Barretto 1947; Martins et al. 1978), Salesópolis (Barretto 1947; Martins et al. 1978), São José dos Campos (Barretto 1947; Martins et al. 1978), São Paulo [F] (Fonseca, 1936)(Barretto 1947; FSP; Martins et al. 1978; Galati 1981); São Roque (Barretto 1947; Martins et al. 1978), Sorocaba (Barretto 1947; Martins et al. 1978), Ubatuba (SUCEN/SP 2006), Una (Ibiúna hoje) (Martins et al. 1978). Obs.: Houve associação errônea entre os sexos de P. arthuri e P. lloydi, ambas descritas apenas pela fêmea (Galati 1981), portanto os identificados como P. arthuri até 1981, possivelmente representem P. lloydi.

lloydi (Antunes, 1937)

Distribuição: Cajamar (SUCEN/SP 2006), Campinas (Barretto 1947; Martins et al. 1978), Cotia (SUCEN/SP 2006), Embu-Guaçu (SUCEN/SP 2006), Francisco Morato (SUCEN/SP 2006), Mairinque (SUCEN/SP 2006; Taniguchi et al. 1990), Mairiporã (SUCEN/ SP 2006), Osasco (Barretto 1947; Martins et al. 1978; MZUSP; Galati 1981), Piedade (Barretto 1947; Martins et al. 1978, Galati 1981), Pirapora do Bom Jesus (SUCEN/SP 2006), Santa Isabel (SUCEN/SP 2006), Salesópolis (Barretto 1947; Martins et al. 1978), São José dos Campos (Barretto 1947; Martins et al. 1978), São Paulo [F] (Antunes, 1937) (Barretto 1947; Martins et al. 1978; FSP), São Roque (Taniguchi et al. 1990; SUCEN/SP 2006), Tietê (Barretto 1947; Martins et al. 1978). Obs.: devido à associação errônea entre os sexos de P. lloydi e de P. arthuri (Galati 1981), machos identificados como de P. lloydi até 1981, possivelmente representem os de P. arthuri. 
ayrozai (Barretto e Coutinho, 1940)

Distribuição: Cajamar (SUCEN/SP 2006), Cananéia (Gomes e Galati 1989; Gomes et al. 1990a), Caraguatatuba (SUCEN/SP 2006), Casa Grande (Salesópolis hoje) (Barretto 1947), Eldorado (Taniguchi et al. 2002), Iporanga (observações não publicadas), Mairiporã (SUCEN/SP 2006), Miracatu (Gomes et al. 1990b), Pariquera-Açú (Gomes e Galati 1987), Pedro de Toledo (Forattini et al. 1976b; Gomes et al. 1990b; Domingos et al. 1998) SUCEN/SP 2006), Ribeirão Grande (Galati et al. 2010), São José dos Campos, (Barretto e Coutinho, 1940), São Paulo [M] (Barretto e Coutinho, 1940) (SUCEN/SP 2006), Ubatuba (Forattini e Galati 1977), Una (Ibiúna hoje) (Barretto 1947; Martins et al. 1978). Obs.: A fêmea de P. guyanensis (Floch \& Abonnenc, 1941) foi erroneamente associada como de $P$. ayrozai, quando da descrição desta espécie (Galati et al. 1977). Estes autores apresentaram a primeira descrição da verdadeira fêmea de P. ayrozai. Segundo Young \& Duncan (1994), P. guyanensis foi descrita por uma única fêmea e, aparentemente, o tipo foi perdido, não se sabendo ao certo se esta fêmea seria a de $P$. geniculatus, $P$. corossoniensis ou de $P$. dorlinsis. Esses autores consideram a espécie de ocorrência no Estado de São Paulo como P. geniculatus. Opinião seguida no presente estudo.

hirsutus (Mangabeira, 1942)

Distribuição: registrada no estado de São Paulo (observações não publicadas)

série guyanensis Barretto, 1962

geniculatus (Mangabeira,1941)

Distribuição: Cananéia (como P. guyanensis, Gomes e Galati 1989), Iporanga (observações não publicadas), Miracatu (como P. guyanensis Forattini et al. 1976a; Gomes et al. 1990b), Pedro de Toledo (como P. guyanensis Forattini et al. 1976; Gomes et al. 1990b), Pedro de Toledo (Forattini et al. 1976a; Domingos et al. 1998; SUCEN/, 2006), Miracatu (como P. guyanensis, Forattini et al. 1976a), Ribeirão Grande (Galati et al. 2010), Santos (como P. guyanensis, Forattini et al. 1976a), Ubatuba (como P. guyanensis, Forattini et al. 1976a), Una (F) (Ibiúna hoje) (como P. ayrozai Barretto e Coutinho, 1940; como P. guyanensis Forattini e Galati, 1977).

Nyssomyia Barretto, 1962

intermedia (Lutz e Neiva, 1912)

Distribuição: Bananal (Marcondes et al. 1998), Barra do Turvo (Andrade Filho et al. 2007), Cananéia (Gomes e Galati, 1989), Caraguatatuba (Marcondes et al. 1998; Condino et al. 2008), Eldorado (Taniguchi et al. 2002), Ilhabela (Andrade Filho et al. 2007; Condino et al. 2008), Iporanga (Galati et al. 2002; Galati et al. 2009), Itariri (Andrade Filho et al. 2007), Juquiá (Andrade Filho et al. 2007), Pariqüera-Açú (Marcondes et al. 1998), Pedro de Toledo (Forattini et al. 1976b; Andrade Filho et al. 2007), Ribeirão Grande (Galati et al. 2010), São Sebastião (Andrade Filho et al. 2007, Marcondes et al. 1998, Brito et al. 2002; Condino et al. 2008), Taubaté (Casanova com. pess.), Ubatuba (Marcondes et al. 1998; Condino et al. 2008).

neivai (Pinto, 1926)

Distribuição: Angatuba (Marcondes et al. 1998), Araçatuba (Marcondes et al. 1998), Araraquara (Marcondes et al. 1998), Atibaia (Marcondes et al. 1998), Barra do Turvo (Andrade Filho et al. 2007), Barueri (Andrade Filho et al. 2007), Caçapava (Andrade Filho et al. 2007), Cajamar (Marcondes et al. 1998), Cajati (Andrade Filho et al. 2007), Capela do Alto (Marcondes et al. 1998), Conchal (Casanova et al. 2005), Dourado (Marcondes et al. 1998; Galati et al. 2002), Eldorado (Marcondes et al. 1998; Andrade Filho et al. 2007), Ipeúna (Cutolo et al. 2008), Iporanga (Marcondes et al. 1998; Galati et al. 2002; Galati et al. 2009), Itariri (Andrade Filho et al. 2007), Itupeva (Marcondes et al. 1998), Juquiá (Andrade Filho et al. 2007), Junqueirópolis (MZUSP), Luis Antônio (Andrade Filho et al. 2007), Miracatu (Marcondes et al. 1998), Mirandópolis (Odorizzi e Galati 2007), Mogi das Cruzes (MZUSP), Monte Aprazível (Marcondes et al. 1998), Natividade da Serra (Marcondes et al. 1998), Osasco (Andrade Filho et al. 2007), Ourinhos (Andrade Filho et al. 2007), Pacaembu (MZUSP), Pariqüera-Açú (Marcondes et al. 1998), Pedro de Toledo (Marcondes et al. 1998), Pereira Barreto (Marcondes et al. 1998), Pirapora do Bom Jesus (Marcondes et al. 1998), Porto Ferreira (Marcondes et al. 1998), Ribeirão Branco (Andrade Filho et al. 2007), Ribeirão Grande (Galati et al. 2010), Ribeirão Preto (MZUSP), São Luís do Paraitinga (Marcondes et al. 1998), São José dos Campos (MZUSP), São Roque (Marcondes et al. 1998), São Paulo (MZUSP), Taubaté (Casanova com. pess.), Teodoro Sampaio (Marcondes et al. 1998).

singularis (Costa Lima, 1932)

Distribuição: Juqueri [F] (Franco da Rocha hoje)(Costa Lima 1932; Barretto 1947; Martins et al. 1978).

whitmani (Antunes e Coutinho, 1939)

Distribuição: Adamantina (SUCEN/SP 2006), Alumínio (SUCEN/SP 2006), Andradina (Barretto 1947; Forattini 1954; Martins et al. 1978), Araçariguama (SUCEN/SP 2006), Araçatuba (Barretto 1947; Martins et al. 1978), Araçoiaba da Serra (SUCEN/SP 2006), Araraquara (Barretto 1947; Martins et al. 1978), Arealva (SUCEN/SP 2006), Assis (Barretto 1947; Martins et al. 1978), Avanhandava (Barretto 1947; Martins et al. 1978), Avaré (Barretto 1947; Martins et al. 1978), Bananal (SUCEN/SP 2006), Barra Bonita (Barretto 1947; Martins et al. 1978), Birigui (Barretto 1947; Martins et al. 1978), Boituva (SUCEN/SP 2006), Caçapava (SUCEN/SP 2006), Cachoeira Paulista (SUCEN/ SP 2006), Cajamar (SUCEN/SP 2006), Capela do Alto (SUCEN/SP 2006), Cássia dos Coqueiros (MZUSP), Catanduva (Barretto 1947; Martins et al. 1978), Chavantes (SUCEN/SP 2006), Corumbataí (Cutolo e Von Zuben 2008), Cunha (SUCEN/SP 2006), Dracena (Forattini 1954), Embu-Guaçu (SUCEN/SP 2006), Gália (SUCEN/SP 2006), Gracinópolis (Forattini 1954), Ibira (SUCEN/SP 2006), Igaratá (SUCEN/SP 2006), Inúbia Paulista (SUCEN/SP 2006), Iperó (SUCEN/SP 2006), Ipeúna (Cutolo et al. 2008), Itabera (SUCEN/SP 2006), Itapetininga (SUCEN/SP 2006), Itaporanga (SUCEN/SP 2006), Itu (SUCEN/SP 2006), Itupeva (Mayo et al. 1998; Pignatti et al. 1995), 
Jambeiro (SUCEN/SP 2006), José Bonifácio (Barretto 1947; Martins et al. 1978), Junqueirópolis (Forattini 1954), Lagoinha (SUCEN/SP 2006), Lins (Barretto 1947; Martins et al. 1978), Mairinque (SUCEN/SP 2006), Marília (Barretto 1947; Martins et al. 1978), Martinópolis (Barretto 1947; Martins et al. 1978), Mirassol (Barretto 1947; Martins et al. 1978), Mogi Mirim (SUCEN/SP 2006), Monte Aprazível (Barretto 1947; Martins et al. 1978), Natividade da Serra (SUCEN/SP 2006), Novo Horizonte (Barretto 1947; Martins et al. 1978), Olímpia (Barretto 1947; Martins et al. 1978), Osvaldo Cruz (SUCEN/SP 2006), Ourinhos (Barretto 1947; Martins et al. 1978), Palmital (Barretto 1947; Martins et al. 1978), Pacaembu (Forattini 1954), Pedreira (SUCEN/SP 2006), Penápolis (Barretto 1947; Martins et al. 1978), Pereira Barreto (Barretto 1947; Martins et al. 1978), Piedade (SUCEN/SP 2006), Pilar do Sul (SUCEN/SP 2006), Pindorama (Barretto 1947; Martins et al. 1978), Piraju (SUCEN/SP 2006), Pirajuí (Barretto 1947; Martins et al. 1978), Pirapora do Bom Jesus (SUCEN/SP 2006), Pirapozinho (Forattini 1954), Platina (SUCEN/SP 2006), Pompéia (Barretto 1947; Martins et al. 1978), Porangaba (SUCEN/SP 2006), Porto Feliz (SUCEN/SP 2006), Presidente Alves (Barretto 1947; Martins et al. 1978), Presidente Epitácio (Forattini 1954), Presidente Prudente (Barretto 1947; Forattini 1954; Martins et al. 1978), Presidente Venceslau (Barretto 1947; Forattini 1954; Martins et al. 1978), Queiróz (MZUSP), Rancharia (Barretto 1947; Martins et al. 1978), Regente Feijó (Barretto 1947; Martins et al. 1978), Ribeira (SUCEN/SP 2006), Riversul (SUCEN/SP 2006), Salto (SUCEN/SP 2006), Salto do Pirapora (SUCEN/SP 2006), Salto Grande (SUCEN/SP 2006), Santa Branca (SUCEN/SP 2006), Santa Cruz do Rio Pardo (SUCEN/SP 2006), Santo Anastácio (Barretto 1947; Forattini 1954; Martins et al. 1978), Santo Antônio do Jardim (SUCEN/SP 2006), São João da Boa Vista (SUCEN, 2006), São José do Rio Preto (Barretto 1947; Martins et al. 1978), São José dos Campos (SUCEN/SP 2006), São Luís do Paraitinga (SUCEN/SP 2006), São Paulo (Barretto 1947; Martins et al. 1978), São Pedro do Turvo (SUCEN/SP 2006), São Roque (SUCEN/SP 2006; Taniguchi et al. 1990), São Sebastião (Brito et al. 2002), São Sebastião da Grama (SUCEN/SP 2006), Silveira (SUCEN/SP 2006), Sorocaba (SUCEN/SP 2006), Tanabi (Barretto 1947; Martins et al. 1978), Tatuí (SUCEN/SP 2006), Tejupa (SUCEN, 2006), Teodoro Sampaio (Condino et al. 1998; SUCEN/SP 2006), Timburi (SUCEN/SP 2006), Tupã (Barretto 1947; Martins et al. 1978; Gomes et al. 1989), Valparaíso (Barretto 1947; Forattini 1954; Martins et al. 1978), Vargem Grande do Sul (SUCEN/SP 2006), Vera Cruz (Barretto 1947; Martins et al. 1978). 
Apêndice 2. Checklist Phlebotominae.

Appendix 2. Checklist of Phlebotominae.

\begin{tabular}{|c|c|c|c|c|c|c|c|c|c|c|}
\hline \multicolumn{5}{|c|}{ Dados da espécie } & \multicolumn{5}{|c|}{ Dados de espécime depositado } & \multirow[t]{2}{*}{ Obs. } \\
\hline Classificação & Gênero & $\begin{array}{c}\text { Epíteto } \\
\text { específico }\end{array}$ & Descritor & $\begin{array}{c}\text { Ano da } \\
\text { descrição }\end{array}$ & $\begin{array}{c}\text { Coleção } \\
\text { científica }\end{array}$ & $\begin{array}{l}\text { Número de } \\
\text { registro }\end{array}$ & Coletor & $\begin{array}{c}\text { Número } \\
\text { do } \\
\text { coletor }\end{array}$ & $\begin{array}{c}\text { É tipo } \\
\text { para a } \\
\text { espécie? }\end{array}$ & \\
\hline $\begin{array}{l}\text { Galati } \\
(2003)\end{array}$ & Brumptomyia & avellari & Costa Lima & 1932 & $\begin{array}{l}\text { Coleção do } \\
\text { Instituto }\end{array}$ & $1421-1424$ & & & Sim & \\
\hline $\begin{array}{l}\text { Galati } \\
(2003)\end{array}$ & Brumptomyia & bragai & $\begin{array}{l}\text { Mangabeira } \\
\& \text { Sherlock }\end{array}$ & 1961 & $\begin{array}{l}\text { Oswaldo Cruz } \\
\text { Núcleo de } \\
\text { Pesquisas da } \\
\text { Bahia e Seção } \\
\text { de Entomologia } \\
\text { do Instituto } \\
\text { Oswaldo Cruz }\end{array}$ & & $\begin{array}{l}\text { Braga, A., } \\
\text { Virgens, D., } \\
\text { Sherlock, I. }\end{array}$ & & Sim & \\
\hline $\begin{array}{l}\text { Galati } \\
(2003)\end{array}$ & Brumptomyia & brumpti & Larrousse & 1920 & $\begin{array}{l}\text { Coleção do } \\
\text { Instituto }\end{array}$ & $1467-1470$ & Brumpt & & Não & \\
\hline $\begin{array}{l}\text { Galati } \\
(2003)\end{array}$ & Brumptomyia & cardosoi & $\begin{array}{c}\text { Barretto \& } \\
\text { Coutinho }\end{array}$ & 1941 & $\begin{array}{c}\text { Oswaldo Cruz } \\
\text { Coleção Padrão } \\
\text { do Departamento } \\
\text { de Parasitologia, } \\
\text { Faculdade de } \\
\text { Medicina/USP }\end{array}$ & 433 & $\begin{array}{l}\text { Barretto, M.P., } \\
\text { Coutinho, J.O. }\end{array}$ & & Sim & \\
\hline $\begin{array}{l}\text { Galati } \\
(2003)\end{array}$ & Brumptomyia & carvalheiroi & $\begin{array}{c}\text { Shimabukuro, } \\
\text { Marassá \& } \\
\text { Galati }\end{array}$ & 2007 & $\begin{array}{l}\text { Coleção do } \\
\text { Departamento de } \\
\text { Epidemiologia, } \\
\text { Faculdade de } \\
\text { Saúde Pública/ } \\
\text { USP }\end{array}$ & & Barretto, M.P. & & Sim & \\
\hline $\begin{array}{l}\text { Galati } \\
(2003)\end{array}$ & Brumptomyia & cunhai & Mangabeira & 1942 & $\begin{array}{c}\text { Coleção Octavio } \\
\text { Mangabeira/ } \\
\text { Intituto Oswaldo } \\
\text { Cruz }\end{array}$ & 215 & Mangabeira, $\mathrm{O}$. & & Sim & Paratipo \\
\hline $\begin{array}{l}\text { Galati } \\
(2003)\end{array}$ & Brumptomyia & galindoi & $\begin{array}{c}\text { Fairchild \& } \\
\text { Hertig }\end{array}$ & 1947 & $\begin{array}{l}\text { Museum of } \\
\text { Comparative } \\
\text { Zoology, } \\
\text { Cambridge, } \\
\text { Mass, USA }\end{array}$ & & Galindo, P. & & Sim & \\
\hline $\begin{array}{l}\text { Galati } \\
(2003)\end{array}$ & Brumptomyia & guimaraesi & $\begin{array}{c}\text { Coutinho \& } \\
\text { Barretto }\end{array}$ & 1941 & $\begin{array}{l}\text { Coleção Padrão } \\
\text { do Departamento } \\
\text { de Parasitologia, } \\
\text { Faculdade de } \\
\text { Medicina/USP }\end{array}$ & 580,581 & $\begin{array}{c}\text { Coutinho, } \\
\text { J.O., Barretto, } \\
\text { M.P. }\end{array}$ & & Sim & \\
\hline $\begin{array}{l}\text { Galati } \\
(2003)\end{array}$ & Brumptomyia & mangabeirai & $\begin{array}{c}\text { Barretto \& } \\
\text { Coutinho }\end{array}$ & 1941 & $\begin{array}{c}\text { Coleção Padrão } \\
\text { do Departamento } \\
\text { de Parasitologia, } \\
\text { Faculdade de } \\
\text { Medicina/USP }\end{array}$ & 444-448 & $\begin{array}{l}\text { Barretto, M.P., } \\
\text { Coutinho, J.O. }\end{array}$ & & Sim & \\
\hline $\begin{array}{l}\text { Galati } \\
(2003)\end{array}$ & Brumptomyia & nitzulescui & Costa Lima & 1932 & $\begin{array}{l}\text { Coleção do } \\
\text { Museu de } \\
\text { Zoologia/USP }\end{array}$ & & & & Não & \\
\hline $\begin{array}{l}\text { Galati } \\
(2003)\end{array}$ & Brumptomyia & ortizi & $\begin{array}{c}\text { Martins, } \\
\text { Silva \& } \\
\text { Falcão }\end{array}$ & 1971 & $\begin{array}{c}\text { Departamento } \\
\text { de Zoologia e } \\
\text { Parasitologia, } \\
\text { Insituto de } \\
\text { Ciências } \\
\text { Biológicas da } \\
\text { UFMG }\end{array}$ & $41225 \mathrm{~A}$ & $\begin{array}{c}\text { Martins, A.V., } \\
\text { Silva, J.E., } \\
\text { Falcão, A.L. }\end{array}$ & & Sim & \\
\hline $\begin{array}{l}\text { Galati } \\
(2003)\end{array}$ & Brumptomyia & pintoi & Costa Lima & 1932 & $\begin{array}{l}\text { Coleção do } \\
\text { Instituto }\end{array}$ & $1425-1427$ & & & Sim & \\
\hline $\begin{array}{l}\text { Galati } \\
(2003)\end{array}$ & Brumptomyia & troglodytes & Lutz & 1922 & $\begin{array}{c}\text { Oswaldo Cruz } \\
\text { Coleção Octavio } \\
\text { Mangabeira/ } \\
\text { Intituto Oswaldo } \\
\text { Cruz }\end{array}$ & $\begin{array}{c}\text { L- 631-633, } \\
635- \\
637.639\end{array}$ & & & Sim & cotipos \\
\hline $\begin{array}{l}\text { Galati } \\
(2003)\end{array}$ & Micropygomyia & ferreirana & $\begin{array}{l}\text { Barretto, } \\
\text { Martins \& } \\
\text { Pellegrino }\end{array}$ & 1956 & $\begin{array}{c}\text { Coleção do } \\
\text { Museu de } \\
\text { Zoologia/USP }\end{array}$ & & $\begin{array}{c}\text { Barretto, M.P., } \\
\text { Martins A.V., } \\
\text { Pellegrino, J. }\end{array}$ & & Sim & $\begin{array}{c}\text { Holótipo e } \\
\text { parátipo }\end{array}$ \\
\hline
\end{tabular}


Apêndice 2. Continuação...

\begin{tabular}{|c|c|c|c|c|c|c|c|c|c|c|}
\hline \multicolumn{5}{|c|}{ Dados da espécie } & \multicolumn{5}{|c|}{ Dados de espécime depositado } & Obs. \\
\hline Classificação & Gênero & $\begin{array}{c}\text { Epíteto } \\
\text { específico }\end{array}$ & Descritor & $\begin{array}{c}\text { Ano da } \\
\text { descrição }\end{array}$ & $\begin{array}{l}\text { Coleção } \\
\text { científica }\end{array}$ & $\begin{array}{l}\text { Número de } \\
\text { registro }\end{array}$ & Coletor & $\begin{array}{c}\text { Número } \\
\text { do } \\
\text { coletor }\end{array}$ & $\begin{array}{c}\text { É tipo } \\
\text { para a } \\
\text { espécie? }\end{array}$ & \\
\hline $\begin{array}{l}\text { Galati } \\
(2003)\end{array}$ & Micropygomyia & longipennis & Barretto & 1946 & $\begin{array}{c}\text { Coleção do } \\
\text { Museu de } \\
\text { Zoologia/USP }\end{array}$ & & Albertin & & Não & \\
\hline $\begin{array}{l}\text { Galati } \\
(2003)\end{array}$ & Micropygomyia & petari & $\begin{array}{l}\text { Galati, } \\
\text { Marassá \& } \\
\text { Andrade }\end{array}$ & 2003 & $\begin{array}{l}\text { Coleção do } \\
\text { Departamento de } \\
\text { Epidemiologia, } \\
\text { Faculdade de } \\
\text { Saúde Pública/ } \\
\text { USP }\end{array}$ & & $\begin{array}{c}\text { Galati, E.A.B, } \\
\text { Marassá, } \\
\text { A.M., } \\
\text { Gonçalves, } \\
\text { R.M., Galati, } \\
\text { A }\end{array}$ & & Sim & \\
\hline $\begin{array}{l}\text { Galati } \\
(2003)\end{array}$ & Micropygomyia & quinquefer & Dyar & 1929 & $\begin{array}{l}\text { U.S. Natural } \\
\text { History Museum }\end{array}$ & 41593 & $\begin{array}{l}\text { Shannon \& } \\
\text { Shannon }\end{array}$ & & Sim & \\
\hline $\begin{array}{l}\text { Galati } \\
(2003)\end{array}$ & Micropygomyia & schreiberi & $\begin{array}{l}\text { Martins, } \\
\text { Falcão \& } \\
\text { Silva }\end{array}$ & 1975 & $\begin{array}{c}\text { Coleção do } \\
\text { Museu de } \\
\text { Zoologia/USP }\end{array}$ & & $\begin{array}{l}\text { Domingos \& } \\
\text { Tomé }\end{array}$ & & Não & \\
\hline $\begin{array}{l}\text { Galati } \\
(2003)\end{array}$ & Sciopemyia & microps & Mangabeira & 1942 & $\begin{array}{l}\text { Coleção Octavio } \\
\text { Mangabeira, } \\
\text { Instituto }\end{array}$ & $\begin{array}{l}\mathrm{L}-436, \\
438-440\end{array}$ & Frutuoso, E. & & Sim & $\begin{array}{l}\text { Holótipo e } \\
\text { parátipo }\end{array}$ \\
\hline $\begin{array}{l}\text { Galati } \\
\text { (2003) }\end{array}$ & Sciopemyia & sordellii & $\begin{array}{l}\text { Shannon \& } \\
\text { Del Ponte }\end{array}$ & 1927 & $\begin{array}{c}\text { Oswaldo Cruz } \\
\text { CRNIF-IRR/ } \\
\text { FIOCRUZ }\end{array}$ & & $\begin{array}{c}\text { Andrade- } \\
\text { Filho, } \\
\text { J.D.,Oliveira, } \\
\text { R.C., } \\
\text { Fonseca, A.R. }\end{array}$ & & Não & \\
\hline $\begin{array}{l}\text { Galati } \\
(2003)\end{array}$ & Lutzomyia & amarali & $\begin{array}{l}\text { Barretto \& } \\
\text { Coutinho }\end{array}$ & 1940 & $\begin{array}{c}\text { Coleção Padrão } \\
\text { do Departamento } \\
\text { de Parasitologia, } \\
\text { Faculdade de } \\
\text { Medicina/USP }\end{array}$ & $352-356$ & $\begin{array}{l}\text { Barretto, M.P., } \\
\text { Coutinho, J.O. }\end{array}$ & & Sim & \\
\hline $\begin{array}{l}\text { Galati } \\
\text { (2003) }\end{array}$ & Lutzomyia & castroi & $\begin{array}{l}\text { Barretto \& } \\
\text { Coutinho }\end{array}$ & 1941 & $\begin{array}{l}\text { Coleção Padrão } \\
\text { do Departamento } \\
\text { de Parasitologia, } \\
\text { Faculdade de } \\
\text { Medicina/USP }\end{array}$ & 489 & $\begin{array}{l}\text { Barretto, M.P., } \\
\text { Coutinho, J.O. }\end{array}$ & & Sim & \\
\hline $\begin{array}{l}\text { Galati } \\
(2003)\end{array}$ & Lutzomyia & almerioi & $\begin{array}{l}\text { Galati \& } \\
\text { Nunes }\end{array}$ & 1999 & $\begin{array}{l}\text { Coleção do } \\
\text { departamento de } \\
\text { Epidemiologia, } \\
\text { Faculdade de } \\
\text { Saúde Pública/ } \\
\text { USP }\end{array}$ & & $\begin{array}{c}\text { Galati, E.A.B } \\
\text { \& Nunes, V. } \\
\text { L.B. }\end{array}$ & & & \\
\hline $\begin{array}{l}\text { Galati } \\
(2003)\end{array}$ & Lutzomyia & dispar & $\begin{array}{l}\text { Martins \& } \\
\text { Silva }\end{array}$ & 1963 & $\begin{array}{l}\text { CRNIF-IRR/ } \\
\text { FIOCRUZ }\end{array}$ & & & & Sim & \\
\hline $\begin{array}{l}\text { Galati } \\
(2003)\end{array}$ & Lutzomyia & longipalpis & Lutz \& Neiva & 1912 & $\begin{array}{l}\text { Coleção do } \\
\text { Museu de } \\
\text { Zoologia/USP }\end{array}$ & & Deane, L.M. & & Não & \\
\hline $\begin{array}{l}\text { Galati } \\
(2003)\end{array}$ & Migonemyia & migonei & França & 1920 & $\begin{array}{l}\text { Coleção do } \\
\text { Departamento de } \\
\text { Epidemiologia, } \\
\text { Faculdade de } \\
\text { Saúde Pública/ } \\
\text { USP }\end{array}$ & & & & Não & \\
\hline $\begin{array}{l}\text { Galati } \\
(2003)\end{array}$ & Migonemyia & rabelloi & $\begin{array}{c}\text { Galati \& } \\
\text { Gomes }\end{array}$ & 1992 & $\begin{array}{l}\text { Coleção do } \\
\text { Departamento de } \\
\text { Epidemiologia, } \\
\text { Faculdade de } \\
\text { Saúde Pública/ } \\
\text { USP }\end{array}$ & & $\begin{array}{c}\text { Galati, E.A.B., } \\
\text { Moraes \& } \\
\text { Oliveira }\end{array}$ & & Sim & \\
\hline $\begin{array}{l}\text { Galati } \\
\text { (2003) }\end{array}$ & Migonemyia & vaniae & $\begin{array}{l}\text { Galati, } \\
\text { Fonseca \& } \\
\text { Marassá }\end{array}$ & 2007 & $\begin{array}{c}\text { Coleção do } \\
\text { Departamento de } \\
\text { Epidemiologia, } \\
\text { Faculdade de } \\
\text { Saúde Pública/ } \\
\text { USP }\end{array}$ & & $\begin{array}{c}\text { Galati, E.A.B, } \\
\text { Fonseca, } \\
\text { M.B., } \\
\text { Andrade, J.R. }\end{array}$ & & Sim & \\
\hline
\end{tabular}


Apêndice 2. Continuação...

\begin{tabular}{|c|c|c|c|c|c|c|c|c|c|c|}
\hline \multicolumn{5}{|c|}{ Dados da espécie } & \multicolumn{5}{|c|}{ Dados de espécime depositado } & \multirow[t]{2}{*}{ Obs. } \\
\hline Classificação & Gênero & $\begin{array}{c}\text { Epíteto } \\
\text { específico }\end{array}$ & Descritor & $\begin{array}{c}\text { Ano da } \\
\text { descrição }\end{array}$ & $\begin{array}{c}\text { Coleção } \\
\text { científica }\end{array}$ & $\begin{array}{l}\text { Número de } \\
\text { registro }\end{array}$ & Coletor & $\begin{array}{c}\begin{array}{c}\text { Número } \\
\text { do } \\
\text { coletor }\end{array} \\
\end{array}$ & $\begin{array}{c}\text { É tipo } \\
\text { para a } \\
\text { espécie? }\end{array}$ & \\
\hline $\begin{array}{l}\text { Galati } \\
(2003)\end{array}$ & Migonemyia & bursiformis & $\begin{array}{c}\text { Floch \& } \\
\text { Abonnenc }\end{array}$ & 1944 & & & & & & \\
\hline $\begin{array}{l}\text { Galati } \\
(2003)\end{array}$ & Pintomyia & bianchigalatiae & $\begin{array}{l}\text { Andrade- } \\
\text { Filho, } \\
\text { Aguiar, Dias } \\
\text { \& Falcão }\end{array}$ & 1999 & $\begin{array}{l}\text { CRNIF-IRR/ } \\
\text { FIOCRUZ }\end{array}$ & 38830 & Leôncio, A & & Sim & \\
\hline $\begin{array}{l}\text { Galati } \\
(2003)\end{array}$ & Pintomyia & christenseni & $\begin{array}{l}\text { Young \& } \\
\text { Duncan }\end{array}$ & 1994 & $\begin{array}{l}\text { CRNIF-IRR/ } \\
\text { FIOCRUZ }\end{array}$ & & $\begin{array}{c}\text { Andrade- } \\
\text { Filho, } \\
\text { J.D.,Oliveira, } \\
\text { R.C., } \\
\text { Fonseca, A.R. }\end{array}$ & & Não & \\
\hline $\begin{array}{l}\text { Galati } \\
\text { (2003) }\end{array}$ & Pintomyia & fischeri & Pinto & 1926 & $\begin{array}{c}\text { Coleção do } \\
\text { Departamento de } \\
\text { Epidemiologia, } \\
\text { Faculdade de } \\
\text { Saúde Pública/ } \\
\text { USP }\end{array}$ & $1812-1817$ & & & Não & \\
\hline $\begin{array}{l}\text { Galati } \\
(2003)\end{array}$ & Pintomyia & pessoai & $\begin{array}{l}\text { Coutinho \& } \\
\text { Barretto }\end{array}$ & 1941 & $\begin{array}{c}\text { Coleção do } \\
\text { Departamento de } \\
\text { Epidemiologia, } \\
\text { Faculdade de } \\
\text { Saúde Pública/ } \\
\text { USP }\end{array}$ & & & & Sim & $\begin{array}{c}\text { macho } \\
\text { holótipo } \\
\text { e fêmea } \\
\text { alótipo }\end{array}$ \\
\hline $\begin{array}{l}\text { Galati } \\
(2003)\end{array}$ & Pintomyia & misionensis & Castro & 1959 & & & & & & \\
\hline $\begin{array}{l}\text { Galati } \\
(2003)\end{array}$ & Pintomyia & monticola & Costa Lima & 1932 & $\begin{array}{c}\text { Coleção do } \\
\text { Departamento de } \\
\text { Epidemiologia, } \\
\text { Faculdade de } \\
\text { Saúde Pública/ } \\
\text { USP }\end{array}$ & 488 & $\begin{array}{l}\text { Barretto, M.P., } \\
\text { Coutinho, J.O. }\end{array}$ & & Sim & $\begin{array}{l}\text { Femea } \\
\text { alótipo }\end{array}$ \\
\hline $\begin{array}{l}\text { Galati } \\
(2003)\end{array}$ & Expapillata & firmatoi & $\begin{array}{l}\text { Barretto, } \\
\text { Martins \& } \\
\text { Pellegrino }\end{array}$ & 1956 & $\begin{array}{l}\text { Departamento } \\
\text { de Parasitologia } \\
\text { da faculdade } \\
\text { de Medicina de } \\
\text { Ribeirão Preto/ } \\
\text { USP }\end{array}$ & & $\begin{array}{c}\text { Barretto, M.P., } \\
\text { Martins A.V., } \\
\text { Pellegrino, J. }\end{array}$ & & Sim & \\
\hline $\begin{array}{l}\text { Galati } \\
(2003)\end{array}$ & Pressatia & choti & $\begin{array}{c}\text { Floch \& } \\
\text { Abonnenc }\end{array}$ & 1941 & $\begin{array}{c}\text { Coleção do } \\
\text { Museu de } \\
\text { Zoologia/USP }\end{array}$ & & $\begin{array}{l}\text { Domingos, } \\
\text { Tomé \& } \\
\text { Afonso }\end{array}$ & & Não & \\
\hline $\begin{array}{l}\text { Galati } \\
(2003)\end{array}$ & Pressatia & trispinosa & Mangabeira & 1942 & $\begin{array}{l}\text { Coleção Adolpho } \\
\text { Lutz, Instituto } \\
\text { Oswaldo Cruz }\end{array}$ & & Mangabeira, $\mathrm{O}$ & & Sim & \\
\hline $\begin{array}{l}\text { Galati } \\
(2003)\end{array}$ & Evandromyia & carmelinoi & $\begin{array}{l}\text { Ryan, Fraiha, } \\
\text { Lainson \& } \\
\text { Shaw }\end{array}$ & 1986 & & & & & & \\
\hline $\begin{array}{l}\text { Galati } \\
(2003)\end{array}$ & Evandromyia & termitophila & $\begin{array}{l}\text { Martins, } \\
\text { Falcão \& } \\
\text { Silva }\end{array}$ & 1964 & $\begin{array}{l}\text { Coleção do } \\
\text { Departamento de } \\
\text { Epidemiologia, } \\
\text { Faculdade de } \\
\text { Saúde Pública/ } \\
\text { USP }\end{array}$ & E-5531 & Frin, A.B. & & Sim & \\
\hline $\begin{array}{l}\text { Galati } \\
(2003)\end{array}$ & Evandromyia & bourrouli & $\begin{array}{c}\text { Barretto \& } \\
\text { Coutinho }\end{array}$ & 1941 & $\begin{array}{c}\text { Coleção do } \\
\text { Museu de } \\
\text { Zoologia/USP }\end{array}$ & & $\begin{array}{l}\text { Barretto, M.P., } \\
\text { Coutinho, J.O. }\end{array}$ & & Não & \\
\hline $\begin{array}{l}\text { Galati } \\
(2003)\end{array}$ & Evandromyia & correalimai & $\begin{array}{l}\text { Martins, } \\
\text { Coutinho \& } \\
\text { Lutz }\end{array}$ & 1970 & $\begin{array}{l}\text { CRNIF-IRR/ } \\
\text { FIOCRUZ }\end{array}$ & & & & & \\
\hline
\end{tabular}


Apêndice 2. Continuação...

\begin{tabular}{|c|c|c|c|c|c|c|c|c|c|c|}
\hline \multicolumn{5}{|c|}{ Dados da espécie } & \multicolumn{5}{|c|}{ Dados de espécime depositado } & \multirow[t]{2}{*}{ Obs. } \\
\hline Classificação & Gênero & $\begin{array}{c}\text { Epíteto } \\
\text { específico }\end{array}$ & Descritor & $\begin{array}{c}\text { Ano da } \\
\text { descrição }\end{array}$ & $\begin{array}{l}\text { Coleção } \\
\text { científica }\end{array}$ & $\begin{array}{l}\text { Número de } \\
\text { registro }\end{array}$ & Coletor & $\begin{array}{l}\text { Número } \\
\text { do } \\
\text { coletor }\end{array}$ & $\begin{array}{c}\text { É tipo } \\
\text { para a } \\
\text { espécie? }\end{array}$ & \\
\hline $\begin{array}{l}\text { Galati } \\
(2003)\end{array}$ & Evandromyia & rupicola & $\begin{array}{l}\text { Martins, } \\
\text { Godoy \& } \\
\text { Silva }\end{array}$ & 1962 & $\begin{array}{l}\text { CRNIF-IRR/ } \\
\text { FIOCRUZ }\end{array}$ & & & & & \\
\hline $\begin{array}{l}\text { Galati } \\
(2003)\end{array}$ & Evandromyia & petropolitana & $\begin{array}{l}\text { Martins \& } \\
\text { Silva }\end{array}$ & 1968 & $\begin{array}{l}\text { CRNIF-IRR/ } \\
\text { FIOCRUZ }\end{array}$ & & & & & \\
\hline $\begin{array}{l}\text { Galati } \\
(2003)\end{array}$ & Evandromyia & cortelezzii & Brèthes & 1923 & $\begin{array}{l}\text { CRNIF-IRR/ } \\
\text { FIOCRUZ }\end{array}$ & & $\begin{array}{c}\text { Andrade- } \\
\text { Filho, } \\
\text { J.D.,Oliveira, } \\
\text { R.C., } \\
\text { Fonseca, A.R. }\end{array}$ & & Não & \\
\hline $\begin{array}{l}\text { Galati } \\
(2003)\end{array}$ & Evandromyia & edwardsi & Mangabeira & 1841 & $\begin{array}{l}\text { Coleção Octavio } \\
\text { Mangabeira/ } \\
\text { Instituto } \\
\text { OswaldoCcruz }\end{array}$ & $\begin{array}{c}\text { L-254, } \\
256-258, \\
260-263, \\
265-269\end{array}$ & & & Sim & $\begin{array}{c}\text { Holótipo e } \\
\text { parátipos }\end{array}$ \\
\hline $\begin{array}{l}\text { Galati } \\
(2003)\end{array}$ & Evandromyia & sallesi & $\begin{array}{l}\text { Galvão \& } \\
\text { Coutinho }\end{array}$ & 1939 & $\begin{array}{l}\text { CRNIF-IRR/ } \\
\text { FIOCRUZ }\end{array}$ & & & & Não & \\
\hline $\begin{array}{l}\text { Galati } \\
(2003)\end{array}$ & Psathyromyia & brasiliensis & Costa Lima & 1932 & $\begin{array}{l}\text { Coleção do } \\
\text { Instituto } \\
\text { Oswaldo Cruz }\end{array}$ & $\begin{array}{l}1383(2), \\
1384(2), \\
1387(2), \\
1388(2)\end{array}$ & & & Sim & \\
\hline $\begin{array}{l}\text { Galati } \\
(2003)\end{array}$ & Psathyromyia & lutziana & Costa Lima & 1932 & $\begin{array}{l}\text { CRNIF-IRR/ } \\
\text { FIOCRUZ }\end{array}$ & $\begin{array}{c}6493, \\
75509 \\
75508\end{array}$ & $\begin{array}{c}\text { Diniz, R., } \\
\text { Andrade- } \\
\text { Filho, J.D., } \\
\text { Brazil, R.P. }\end{array}$ & & Sim & \\
\hline $\begin{array}{l}\text { Galati } \\
\text { (2003) }\end{array}$ & Psathyromyia & pascalei & $\begin{array}{l}\text { Coutinho \& } \\
\text { Barretto }\end{array}$ & 1940 & $\begin{array}{l}\text { Coleção do } \\
\text { Departamento de } \\
\text { Epidemiologia, } \\
\text { Faculdade de } \\
\text { Saúde Pública/ } \\
\text { USP }\end{array}$ & $365-368$ & $\begin{array}{l}\text { Coutinho, } \\
\text { J.O., Barretto, } \\
\text { M.P. }\end{array}$ & & Sim & $\begin{array}{c}3 \text { machos } \\
\text { cotipos }\end{array}$ \\
\hline $\begin{array}{l}\text { Galati } \\
\text { (2003) }\end{array}$ & Psathyromyia & hermanlenti & $\begin{array}{l}\text { Martins, } \\
\text { Silva \& } \\
\text { Falcão }\end{array}$ & 1970 & $\begin{array}{l}\text { CRNIF-IRR/ } \\
\text { FIOCRUZ }\end{array}$ & & & & & \\
\hline $\begin{array}{l}\text { Galati } \\
(2003)\end{array}$ & Psathyromyia & lanei & $\begin{array}{l}\text { Barretto \& } \\
\text { Coutinho }\end{array}$ & 1941 & $\begin{array}{c}\text { Coleção do } \\
\text { Departamento de } \\
\text { Epidemiologia, } \\
\text { Faculdade de } \\
\text { Saúde Pública/ } \\
\text { USP }\end{array}$ & & $\begin{array}{l}\text { Barretto, M.P., } \\
\text { Coutinho, J.O. }\end{array}$ & & Sim & $\begin{array}{c}2 \text { machos } \\
\text { cotipos e } \\
1 \text { fêmea } \\
\text { alotipo }\end{array}$ \\
\hline $\begin{array}{l}\text { Galati } \\
(2003)\end{array}$ & Psathyromyia & pelloni & $\begin{array}{l}\text { Sherlock \& } \\
\text { Alencar }\end{array}$ & 1959 & $\begin{array}{l}\text { Coleção do } \\
\text { Departamento de } \\
\text { Epidemiologia, } \\
\text { Faculdade de } \\
\text { Saúde Pública/ } \\
\text { USP }\end{array}$ & & & & Sim & $\begin{array}{l}1 \text { macho } \\
\text { holótipo }\end{array}$ \\
\hline $\begin{array}{l}\text { Galati } \\
(2003)\end{array}$ & Psathyromyia & pestanai & $\begin{array}{l}\text { Barretto \& } \\
\text { Coutinho }\end{array}$ & 1941 & $\begin{array}{c}\text { Coleção do } \\
\text { Departamento de } \\
\text { Epidemiologia, } \\
\text { Faculdade de } \\
\text { Saúde Pública/ } \\
\text { USP }\end{array}$ & & $\begin{array}{l}\text { Coutinho, } \\
\text { J.O., Barretto, } \\
\text { M.P. }\end{array}$ & & Sim & $\begin{array}{l}1 \text { macho } \\
\text { holótipo } \\
\text { e fêmea } \\
\text { alótipo }\end{array}$ \\
\hline
\end{tabular}


Apêndice 2. Continuação...

\begin{tabular}{|c|c|c|c|c|c|c|c|c|c|c|}
\hline \multicolumn{4}{|c|}{ Dados da espécie } & \multicolumn{6}{|c|}{ Dados de espécime depositado } & \multirow[t]{2}{*}{ Obs. } \\
\hline Classificação & Gênero & $\begin{array}{c}\text { Epíteto } \\
\text { específico }\end{array}$ & Descritor & $\begin{array}{c}\text { Ano da } \\
\text { descrição }\end{array}$ & $\begin{array}{c}\text { Coleção } \\
\text { científica }\end{array}$ & $\begin{array}{l}\text { Número de } \\
\text { registro }\end{array}$ & Coletor & $\begin{array}{c}\begin{array}{c}\text { Número } \\
\text { do } \\
\text { coletor }\end{array} \\
\end{array}$ & $\begin{array}{c}\text { É tipo } \\
\text { para a } \\
\text { espécie? }\end{array}$ & \\
\hline $\begin{array}{l}\text { Galati } \\
(2003)\end{array}$ & Psathyromyia & punctigeniculata & $\begin{array}{c}\text { Floch \& } \\
\text { Abonnenc }\end{array}$ & 1944 & $\begin{array}{c}\text { Coleção do } \\
\text { Departamento de } \\
\text { Epidemiologia, } \\
\text { Faculdade de } \\
\text { Saúde Pública/ } \\
\text { USP }\end{array}$ & $\begin{array}{l}10800, \\
10801\end{array}$ & & & Não & \\
\hline $\begin{array}{l}\text { Galati } \\
(2003)\end{array}$ & Psathyromyia & shannoni & Dyar & 1929 & $\begin{array}{c}\text { Coleção do } \\
\text { Museu de } \\
\text { Zoologia/USP }\end{array}$ & & Coutinho, J.O. & & Não & \\
\hline $\begin{array}{l}\text { Galati } \\
(2003)\end{array}$ & Martinsmyia & alphabetica & Fonseca & 1936 & $\begin{array}{c}\text { Coleção Padrão } \\
\text { do Departamento } \\
\text { de Parasitologia, } \\
\text { Faculdade de } \\
\text { Medicina/USP }\end{array}$ & 369 & $\begin{array}{l}\text { Coutinho, } \\
\text { J.O., Barretto, } \\
\text { M.P. }\end{array}$ & & Sim & \\
\hline $\begin{array}{l}\text { Galati } \\
(2003)\end{array}$ & Bichromomyia & flaviscutellata & Mangabeira & 1943 & $\begin{array}{c}\text { Coleção Octavio } \\
\text { Mangabeira, } \\
\text { Insituto Oswaldo } \\
\text { Cruz }\end{array}$ & $\begin{array}{l}\text { L } 289, \\
\text { L-290 }\end{array}$ & & & Sim & $\begin{array}{l}\text { Alótipo e } \\
\text { parátipo }\end{array}$ \\
\hline $\begin{array}{l}\text { Galati } \\
(2003)\end{array}$ & Psychodopygus & arthuri & Fonseca & 1936 & $\begin{array}{l}\text { Coleção de } \\
\text { Entomologia( } \\
\text { Diptera) do } \\
\text { Instituto } \\
\text { Butantan }\end{array}$ & & & & Sim & $\begin{array}{c}10 \text { fêmeas } \\
\text { metatipos e } \\
2 \text { cotipos }\end{array}$ \\
\hline $\begin{array}{l}\text { Galati } \\
(2003)\end{array}$ & Psychodopygus & lloydi & Antunes & 1937 & $\begin{array}{l}\text { Coleção John } \\
\text { Lane (Faculdade } \\
\text { de Saúde } \\
\text { Pública/USP }\end{array}$ & & & & Sim & $\begin{array}{c}\text { 1fêmea } \\
\text { Holótipo e } \\
3 \text { fêmeas } \\
\text { Parátipos }\end{array}$ \\
\hline $\begin{array}{l}\text { Galati } \\
(2003)\end{array}$ & Psychodopygus & ayrozai & $\begin{array}{c}\text { Barretto \& } \\
\text { Coutinho }\end{array}$ & 1940 & $\begin{array}{c}\text { Coelção do } \\
\text { departamento de } \\
\text { Epidemiologa, } \\
\text { Faculdade de } \\
\text { Saúde Pública/ } \\
\text { USP }\end{array}$ & $\begin{array}{c}357-361 \\
698\end{array}$ & $\begin{array}{l}\text { Barretto, M.P., } \\
\text { Coutinho, J.O. }\end{array}$ & & Sim & $\begin{array}{c}4 \text { machos } \\
\text { cotipos, } 1 \\
\text { f alótipo } \\
\text { (= } \mathrm{P} . \\
\text { geniculatus) }\end{array}$ \\
\hline $\begin{array}{l}\text { Galati } \\
(2003)\end{array}$ & Psychodopygus & hirsutus & Mangabeira & 1942 & $\begin{array}{l}\text { Coleção Adolpho } \\
\text { Lutz, Instituto } \\
\text { Oswaldo Cruz }\end{array}$ & & $\begin{array}{l}\text { Comissão de } \\
\text { Estudos de } \\
\text { Leishmaiose } \\
\text { Visceral } \\
\text { Americana }\end{array}$ & & Sim & \\
\hline $\begin{array}{l}\text { Galati } \\
(2003)\end{array}$ & Psychodopygus & geniculatus & Mangabeira & 1941 & $\begin{array}{c}\text { Coleção Octavio } \\
\text { Mangabeira/ } \\
\text { Intituto Oswaldo } \\
\text { Cruz }\end{array}$ & L-405 e 406 & & & Sim & $\begin{array}{c}\text { Holótipo } \\
\text { e parátipo } \\
\text { (Lâminas } \\
\text { não } \\
\text { existentes) }\end{array}$ \\
\hline $\begin{array}{l}\text { Galati } \\
(2003)\end{array}$ & Nyssomyia & intermedia & Lutz \& Neiva & 1912 & $\begin{array}{c}\text { CRNIF-IRR/ } \\
\text { FIOCRUZ }\end{array}$ & & $\begin{array}{l}\text { Andrade- } \\
\text { Filho, J.D. }\end{array}$ & & Não & \\
\hline $\begin{array}{l}\text { Galati } \\
(2003)\end{array}$ & Nyssomyia & neivai & Pinto & 1926 & $\begin{array}{c}\text { Coleção do } \\
\text { Museu de } \\
\text { Zoologia/USP }\end{array}$ & & & & Não & \\
\hline $\begin{array}{l}\text { Galati } \\
(2003)\end{array}$ & Nyssomyia & singularis & Costa Lima & 1932 & & & & & & \\
\hline $\begin{array}{l}\text { Galati } \\
(2003)\end{array}$ & Nyssomyia & whitmani & $\begin{array}{l}\text { Antunes \& } \\
\text { coutinho, }\end{array}$ & 1939 & $\begin{array}{l}\text { Coleção John } \\
\text { Lane (Faculdade } \\
\text { de Saúde } \\
\text { Pública/USP }\end{array}$ & & & & Sim & $\begin{array}{l}\text { Macho } \\
\text { Holótipo, } \\
\text { Alótipo, } \\
\text { 2M, 2 F } \\
\text { parátipos }\end{array}$ \\
\hline
\end{tabular}

